\title{
DESIGN-DEPENDENT LOADS IN TOPOLOGY OPTIMIZATION
}

\author{
Blaise Bourdin $^{1}$ And Antonin Chambolle ${ }^{2}$
}

\begin{abstract}
We present, analyze, and implement a new method for the design of the stiffest structure subject to a pressure load or a given field of internal forces. Our structure is represented as a subset $S$ of a reference domain, and the complement of $S$ is made of two other "phases", the "void" and a fictitious "liquid" that exerts a pressure force on its interface with the solid structure. The problem we consider is to minimize the compliance of the structure $S$, which is the total work of the pressure and internal forces at the equilibrium displacement. In order to prevent from homogenization we add a penalization on the perimeter of $S$. We propose an approximation of our problem in the framework of $\Gamma$-convergence, based on an approximation of our three phases by a smooth phase-field. We detail the numerical implementation of the approximate energies and show a few experiments.
\end{abstract}

Mathematics Subject Classification. 49Q20, 74P05, 74P15.

Received November 20, 2001. Revised May 13, 2002.

\section{INTRODUCTION}

Topology optimization is a rigorous framework for the design of structures with optimal properties. This method is very general and many different applications have been imagined, see for instance [8]. However, most of the theoretical literature deals with the so-called "minimum compliance" problem, that is, how to design the stiffest (or least compliant) structure under a given fixed load (refer to [30] and [29] for the pioneering works, to [2] for an exhaustive study of the homogenization method or, again, to [8]). A limitation of these approaches comes from the fact that the loads are supposed to be given and cannot depend on the object to be designed itself. For instance, the design of pressurized structure likes dams, pipelines or containers where the loads are a function of the design is not within the reach of these methods. Similarly, they do not allow for the design of structures under gravity forces, since in these situations the application point of the force, that is, the structure itself, is the unknown of the problem.

As the theoretical and numerical tools for the "classical" problems seem now to have reached maturity, the interest tends to shift toward topology optimization under design-dependent loads, as in the examples mentioned above. For instance, in [28] and [15] numerical techniques that deal with the pressure loads are presented.

In this paper, we address two types of design-dependent loads for the minimum compliance: a pressure load, which depends on the shape and the topology of the unknown structure and a volume load like a gravity force, for instance. Our goal is to give a rigorous modeling, a mathematical study and a numerical implementation

Keywords and phrases: Topology optimization, optimal design, design-dependent loads, $\Gamma$-convergence, diffuse interface method.

1 Courant Institute of Mathematical Science, New York University. Now at: Department of Mathematics, Louisiana State University, Baton Rouge LA 70803-4918, USA; e-mail: bourdin@math.1su.edu

2 CEREMADE, UMR 7534 du CNRS, Université de Paris-Dauphine, 75775 Paris Cedex 16, France;

e-mail: chambolle@ceremade.dauphine.fr 
of this problem. The basic ingredients are the "perimeter penalization method" and the idea of variational approximation by $\Gamma$-convergence. The former, detailed in [27] and analyzed in [4] allows for the well-posedness of the problem but is also what makes our numerical implementation possible. The latter, which is also closely related to the "phase field" or "diffuse interface" methods in material science has been used by both authors for various problems related to image processing, fracture mechanics or epitaxially strained crystalline thin films (see $[10-12,14]$ ). An alternate approach for some similar problems has been studied by Osher and Santosa in [36]. In order to achieve some properties of the natural vibration mode of a drum, they consider a topology optimization problem with a perimeter penalization. They represent the unknown structure as the level set of a function. The evolution of this function towards the optimum is governed, in part, by the mean curvature motion equation, which corresponds to the gradient flow of the surface energy in our method. See also [38] for another approach, also based on level sets.

This paper is organized as follows: in Section 1, we give a mathematical formulation of our problem and introduce a weak formulation, where the design is represented by a phase indicator. Then, we present a two step approximation scheme based on a "fictitious material" (in Th. 1.3) and a "phase-field" (in Th. 1.5) model. In Section 2, we detail the numerical implementation and present several experiments. Section 3 is devoted to the proof of the convergence of the phase-field approximation and Section 4 to that of the fictitious material problem. The short Section 5 investigates some properties of the interfaces, in the two-dimensional case only. Lastly, in the appendices, some useful properties of the $\Gamma$-convergence are recalled and two technical lemmas are proved.

\section{Statement of the PRoblem AND of the MAin RESUlts}

\subsection{Notations and statement of the ideal problem}

In the following, we consider an open bounded domain $\Omega \subset \mathbb{R}^{N}$, with Lipschitz-regular boundary, where the optimization problem will take place. In practice, $N=2$ or 3 , but except when otherwise stated most results of this paper are valid in any dimension. In this domain, three "phases" are to be distributed: the structure or "solid" phase, i.e., a part $S \subset \Omega$ occupied by some elastic material, the "liquid" $L$, and some "void" $V$, filling the remaining of $\Omega$. S, $L, V$ thus form a partition of $\Omega$. At each point of $x \in \Omega$, we define a function $p: \bar{\Omega} \rightarrow \mathbb{R}$, the value of the pressure field if some liquid is present. Since we do not know a priori the set $L$, we have to define $p$ in the whole domain $\Omega$, even if it has a physical effect only at the interface $\partial L$. Similarly, we define a force field $f: \bar{\Omega} \rightarrow \mathbb{R}^{N}$ that is to be applied at each point of the structure. We assume that $f$ and $p$ are smooth and that $p$ does not vanish in $\bar{\Omega}$.

The equilibrium displacement associated with a given repartition of the three phases is the minimizer of

$$
\begin{aligned}
E(\{S, L, V\}, u)= & \frac{1}{2} \int_{S} A \mathrm{e}(u)(x): \mathrm{e}(u)(x) \mathrm{d} x-\int_{S} f(x) \cdot u(x) \mathrm{d} x \\
& -\int_{\partial L \cap \Omega} p(x) u(x) \cdot \nu_{L}(x) \mathrm{d} \mathcal{H}^{N-1}(x)
\end{aligned}
$$

among all kinematically admissible displacements. Here, A, the Hooke's law of the linear elastic material we consider, is given for any symmetrical matrix of order $N, \xi$ by $A \xi=(\kappa-2 \mu / N) \operatorname{tr} \xi \mathbf{I}+2 \mu \xi$, where $\kappa$ and $\mu$ are the bulk and shear moduli. The linearized strain is defined as $\mathrm{e}(u)=\left(\nabla u+\nabla u^{t}\right) / 2, \nu_{L}(x)$ is the outer normal to the set $L$ at $x$, and $\mathcal{H}^{N-1}$ is the $(N-1)$-dimensional Hausdorff measure (see for instance $\left.[22,24,25]\right)$. The term $-\int_{\partial L \cap \Omega} p u \cdot \nu_{L} \mathrm{~d} \mathcal{H}^{N-1}$ corresponds to the potential energy of the hydrostatic pressure at the boundary of the liquid, applied to the other phases.

The set $X_{u}$ of admissible displacements consists in the functions $u \in H^{1}\left(\Omega ; \mathbb{R}^{N}\right)$ such that $u=0$ on some (non-negligible) part of the boundary $\partial \Omega$ and $u \cdot \nu_{\Omega}=0$ on some other part (with $\nu_{\Omega}$ the outer normal to $\Omega$, defined $\mathcal{H}^{N-1}$-a.e. since $\partial \Omega$ is Lipschitz). We call $\Gamma^{u}$ the part of $\partial \Omega$ where either one or the other of these 
homogeneous Dirichlet boundary conditions is enforced. Without loss of generality we will consider in the sequel that $u=0$ on all of $\Gamma^{u}$, in order to simplify the notations.

Then, the minimizer $u^{*}$ of (1), if it exists, is the solution of the following weak problem: $u^{*} \in X_{u}$ and for each $v \in X_{u}$,

$$
\int_{S} A \mathrm{e}\left(u^{*}\right)(x): \mathrm{e}(v)(x) \mathrm{d} x=\int_{S} f(x) \cdot v(x) \mathrm{d} x+\int_{\partial L \cap \Omega} p(x) v(x) \cdot \nu_{L}(x) \mathrm{d} \mathcal{H}^{N-1}(x) .
$$

In this case, we can define the compliance of the structure $S$ under the load of pressure field defined in $V$ as the work of the forces at equilibrium, namely

$$
C(S, L, V)=\int_{S} f(x) \cdot u^{*}(x) \mathrm{d} x+\int_{\partial L \cap \Omega} p(x) u^{*}(x) \cdot \nu_{L}(x) \mathrm{d} \mathcal{H}^{N-1}(x)
$$

By choosing $v=u^{*}$ in (2), one recovers the well-known identity

$$
C(S, L, V)=-2 E\left(\{S, L, V\}, u^{*}\right)=-2 \inf _{u \in X_{u}} E(\{S, L, V\}, u)
$$

In particular, the last equality makes possible to define the compliance $C$ for any partition $S, L, V$ of $\Omega$, even when the existence of a minimizer $u^{*}$ is not granted.

Remark 1.1. At each point of the domain, the magnitude of the pressure field is supposed to be known a priori and does not depend on the deformation of the structure. This simplification might seem a little rough but actually makes sense if the part $L$ of the domain is supposed to be connected to a large "container" or caused by some external phenomena. For instance, the pressure of the water in an artificial lake does not vary with the deformation of the dam closing it. In the numerical experiments presented in Section 2.3, we have tried to solve problems where this hypothesis is realistic.

The problem that we will consider in this paper is a generalization of the usual minimum compliance problem, that is, find the configuration $S, L, V$ of given volume fraction, that minimizes $C$.

Unfortunately, as is usual in optimal design, it is unlikely that this problem admits a solution. Indeed, in the absence of strong compactness of the minimizing sequences of designs, the optimal state should be attained by a fine mixture of all three phases. The rigorous framework for studying this problem would then be the theory of homogenization as in $[2,3,17,18]$. However, in our case, it is not clear how to apply this method. Also, the mechanical interpretation of a fine mixture of the "liquid" and some other phase (either "solid" or "void") is not obvious: where would the pressure actually be applied, in what direction? For these reasons, we prefer to consider a slight modification of the problem for which one can prove the existence of solutions of more "classical" interpretation.

We choose (as many others, see for instance [4,27]) to perturb our functional $C$ by adding to it another term that penalizes the total length (or surface) of the interfaces between the three phases, so that our problem becomes

$$
\min _{S, L, V} C(S, L, V)+\lambda \Lambda(S, L, V)
$$

where $\lambda>0$ is a fixed parameter, and

$$
\Lambda(S, L, V)=\mathcal{H}^{N-1}(\partial L \cap \Omega)+\mathcal{H}^{N-1}(\partial V \cap \Omega) .
$$

The energy $\Lambda$ is not symmetric in $S, L$ and $V$. This is because of the underlying assumption that in the optimal configuration there will be no contact in between the liquid and the void. If this is true, the only interfaces we expect to encounter are $\partial S \cap \partial L \cap \Omega$ and $\partial S \cap \partial V \cap \Omega$, that is $\partial S \cap \Omega$, which is exactly measured by $\Lambda$. Otherwise, the surface of $\partial L \cap \partial V \cap \Omega$ is counted twice in $\Lambda$. 
Formally, our assumption is quite intuitive: along an interface $\partial L \cap \partial V$, the would opposes no resistance to the pressure field and therefore should be sent to infinity, leading to an infinite compliance. However, a rigorous approach of this delicate point is much more complicated than this heuristic argument and is addressed (with a complete solution in the 2-dimensional case only) in Section 5.

This choice of $\Lambda$ is very valuable in the numerical implementation: it can be represented through a scalar phase-field approximation (see Sect. 1.4), whereas more symmetric penalizations of the interfaces would require a vector-valued representation.

Problem (3) has to be solved under two additional constraints. First, we want to prescribe the proportion $\theta_{S}, \theta_{L}, \theta_{V} \in(0,1)\left(\theta_{S}+\theta_{L}+\theta_{V}=1\right)$ of each phase by enforcing the constraints $|S|=\theta_{S}|\Omega|,|L|=\theta_{V}|\Omega|$, etc. Then, we also want to consider some "Dirichlet" boundary condition for the partition $S, L, V$. This means that we will consider three disjoint (closed, regular) subsets $\Gamma_{S}, \Gamma_{L}, \Gamma_{V}$ of $\partial \Omega$, that we want to belong, respectively, to $\bar{S}, \bar{L}, \bar{V}$. All the results we show will be valid under the following technical hypothesis:

(H) $\partial \Omega=\Gamma^{u} \cup\left(\Gamma_{S} \cup \Gamma_{L} \cup \Gamma_{V}\right)$.

This means that we need to assign some Dirichlet condition either to $u$, or to the partition $S, L, V$ everywhere on the boundary. In fact, this could be a little relaxed: all results would still hold if we assumed that, everywhere on $\partial \Omega \backslash \Gamma^{u} \cup \Gamma_{L}$, the phase $L$ does not touch the boundary - but in order to simplify the notations we leave this adaption to the reader.

\subsection{The weak formulation}

In all that has been written above we have implicitly assumed that the sets $S, L, V$ have regular topological boundaries $\partial S, \partial L, \partial V$ of finite $\mathcal{H}^{N-1}$ measure. In this setting, again, under the constraints stated above, we cannot ensure that our problem (3) has a solution. However, we may now introduce a weak version of (3) that actually has a solution (that we will then be able to approximate numerically), and that coincides with (3) for smooth partitions. We now consider that $S, L, V$ is a partition of $\Omega$ into three finite-perimeter (or Caccioppoli) sets. A set $E$ of finite perimeter in $\Omega$ is by definition a set whose characteristic function $\mathbf{1}_{E}$ belongs to $B V(\Omega)$, the space of functions with bounded variation in $\Omega$. The reader who is not familiar with this theory may consult for instance $[22,25,26,40]$.

A set of finite perimeter $E$ has a generalized boundary in $\Omega$ called measure-theoretical boundary and denoted by $\partial_{\star} E$. It is the set of points of $\Omega$ where $E$ is not of Lebesgue-density 0 or 1 :

$$
\partial_{\star} E=\left\{x \in \Omega: \limsup _{\rho \downarrow 0} \frac{|E \cap B(x, \rho)|}{\omega_{N} \rho^{N}}>0 \text { and } \liminf _{\rho \downarrow 0} \frac{|E \cap B(x, \rho)|}{\omega_{N} \rho^{N}}<1\right\}
$$

with $\omega_{N}=|B(0,1)|$ the volume of the unit ball in $\mathbb{R}^{N}$. The distributional derivative of $\mathbf{1}_{E}$ in $\Omega$, which by definition is a bounded Radon measure, is carried by the set $\partial_{\star} E$ and given by

$$
D \mathbf{1}_{E}=-\nu_{E} \mathcal{H}^{N-1}\left\llcorner\partial_{\star} E\right.
$$

where $\nu_{E}$ is the generalized outer unit normal to $E$, defined $\mathcal{H}^{N-1}$-a.e. in $\partial_{\star} E$. The perimeter of $E$ in $\Omega$ is the total variation of this measure: $\operatorname{Per}(E, \Omega)=\left|D \mathbf{1}_{E}\right|(\Omega)=\mathcal{H}^{N-1}\left(\partial_{\star} E\right)$. When $E$ is a smooth set, then all these notions coincide with the classical notions $\left(\partial_{\star} E=\partial E \cap \Omega, \nu_{E}\right.$ is the classical outer normal to $\left.E\right)$.

Our case is slightly more complicated. Indeed, we want our set $S$ to be attached in some sense to the boundary $\Gamma_{S}, L$ to $\Gamma_{L}$, and $V$ to $\Gamma_{V}$. However, if we impose for instance that the trace of $\mathbf{1}_{S}$ on $\partial \Omega$ is 1 a.e. on $\Gamma_{S}$, then this property is not stable in the $L^{1}$ topology (the natural topology for the convergence of finiteperimeter sets): if $S_{n} \rightarrow S$ in the sense that $\mathbf{1}_{S_{n}} \rightarrow \mathbf{1}_{S}$ in $L^{1}(\Omega)$ as $n \rightarrow \infty$, and $\mathbf{1}_{S_{n}}=1$ on $\Gamma_{S}$ for every $n$, the trace of $\mathbf{1}_{S}$ on $\Gamma_{S}$ does not have to be 1 as well. In order to take into account this phenomenon we will extend the $B V$ functions $\mathbf{1}_{S}, \mathbf{1}_{L}, \mathbf{1}_{V}$ to $\Omega \cup \Gamma^{\rho}$ (where $\Gamma^{\rho}=\Gamma_{S} \cup \Gamma_{L} \cup \Gamma_{V}$, the notation $\Gamma^{\rho}$ will be made clear in the sequel) by assigning them a fictitious "exterior trace" on $\Gamma^{\rho}$. 
More precisely, we introduce the space $B V\left(\Omega \cup \Gamma^{\rho}\right)$ of functions with bounded variation on $\Omega \cup \Gamma^{\rho}$. Such functions $w$ have an inner trace $w^{\text {int }}$ on $\partial \Omega$, which is the standard trace of $w$ seen as functions in $B V(\Omega)$ (well defined in $L^{1}(\partial \Omega)$ since $\partial \Omega$ is Lipschitz-regular), and an outer trace $w^{\text {ext }} \in L^{1}\left(\Gamma^{\rho}\right)$ on $\Gamma^{\rho} 3$. Their derivatives $D w$ are measures on $\Omega \cup \Gamma^{\rho}$, defined by

$$
\int_{\Omega \cup \Gamma^{\rho}} \varphi(x) \cdot D w(x)=\int_{\Gamma^{\rho}} w^{\operatorname{ext}}(x) \varphi(x) \cdot \nu_{\Omega}(x) \mathrm{d} \mathcal{H}^{N-1}(x)-\int_{\Omega} w(x) \operatorname{div} \varphi(x) \mathrm{d} x
$$

for every $\varphi \in C^{1}\left(\Omega \cup \Gamma^{\rho} ; \mathbb{R}^{N}\right)$ with compact support in $\Omega \cup \Gamma^{\rho}$. In other words, the measure $D w$ is

$$
D w=\left(w^{\text {ext }}-w^{\text {int }}\right) \nu_{\Omega} \mathcal{H}^{N-1}\left\llcorner\Gamma^{\rho}+D w\llcorner\Omega\right.
$$

with $D w\llcorner\Omega$ the classical distributional derivative of $w$ seen as a function in $B V(\Omega)$, and the total variation

$$
|D w|\left(\Omega \cup \Gamma^{\rho}\right)=\int_{\Gamma^{\rho}}\left|w^{\text {ext }}(x)-w^{\text {int }}(x)\right| \mathrm{d} \mathcal{H}^{N-1}(x)+|D w|(\Omega) .
$$

Then, a set $E$ of finite perimeter in $\Omega \cup \Gamma^{\rho}$ is a set $E$ such that $\left|D \mathbf{1}_{E}\right|(\Omega)<+\infty$, together with an outer trace $\mathbf{1}_{E}^{\text {ext }} \in\{0,1\}$ on $\Gamma^{\rho}$ (that can be the characteristic function of an arbitrary measurable subset of $\Gamma^{\rho}$, see [26] (Prop. 2.15)). We introduce the extended measure theoretical boundary

$$
\bar{\partial}_{\star} E=\partial_{\star} E \cup\left\{x \in \Gamma^{\rho}: \mathbf{1}_{E}^{\mathrm{ext}}(x) \neq \mathbf{1}_{E}^{\mathrm{int}}(x)\right\} \subset \Omega \cup \Gamma^{\rho} .
$$

Again, the measure $D \mathbf{1}_{E}$ is carried by $\bar{\partial}_{\star} E$ and given by

$$
D \mathbf{1}_{E}=-\nu_{E} \mathcal{H}^{N-1}\left\llcorner\bar{\partial}_{\star} E\right.
$$

where for every $x \in \Gamma^{\rho}, \nu_{E}(x)=\nu_{\Omega}(x)$ if $\mathbf{1}_{E}^{\text {ext }}=0, \mathbf{1}_{E}^{\text {int }}=1$ and $\nu_{E}(x)=-\nu_{\Omega}(x)$ if $\mathbf{1}_{E}^{\text {ext }}=1, \mathbf{1}_{E}^{\text {int }}=0$. Again, we have $\left|D \mathbf{1}_{E}\right|\left(\Omega \cup \Gamma^{\rho}\right)=\mathcal{H}^{N-1}\left(\bar{\partial}_{\star} E\right)$.

In this setting, we consider $S, L, V$ as finite-perimeter sets in $\Omega \cup \Gamma^{\rho}$, letting $\mathbf{1}_{S}^{\text {ext }}=1$ on $\Gamma_{S}$ and 0 on $\Gamma_{L} \cup \Gamma_{V}$, $\mathbf{1}_{L}^{\mathrm{ext}}=1$ on $\Gamma_{L}$ and 0 on $\Gamma_{S} \cup \Gamma_{V}$, and $\mathbf{1}_{V}^{\text {ext }}=1-\mathbf{1}_{S}^{\text {ext }}-\mathbf{1}_{L}^{\text {ext }}$. For instance, $\bar{\partial}_{\star} L=\partial_{\star} L \cup\left\{x \in \Gamma_{L}: \mathbf{1}_{L}^{\text {int }}=\right.$ $0\} \cup\left\{x \in \Gamma_{S} \cup \Gamma_{V}: \mathbf{1}_{L}^{\text {int }}=1\right\}$.

Then, the correct definition for the penalization is

$$
\bar{\Lambda}(S, L, V)=\mathcal{H}^{N-1}\left(\bar{\partial}_{\star} L\right)+\mathcal{H}^{N-1}\left(\bar{\partial}_{\star} V\right) .
$$

In fact, it is exactly the relaxation (or lower semicontinuous envelope) in $L^{1}(\Omega)$ of the function $\Lambda$ defined by (4) for partitions into regular sets $S, L, V$ with $\Gamma_{S} \subset \bar{S}, \Gamma_{L} \subset \bar{L}, \Gamma_{V} \subset \bar{V}$, and set to $+\infty$ for any other partition. This fact is discussed in Appendix B.

Then, we define again the compliance by

$$
C(S, L, V)=-2 \inf _{u \in X_{u}} E(\{S, L, V\}, u)
$$

\footnotetext{
${ }^{3}$ This space can be precisely defined as the set of the restrictions to $\Omega \cup \Gamma^{\rho}$ of the functions $u \in B V\left(\Omega^{\prime}\right)$, where $\Omega^{\prime}$ is a larger set such that $\Omega^{\prime} \cap \bar{\Omega}$ is $\Omega \cup \Gamma^{\rho}$ (minus the $N-2$ dimensional boundary of the $N-1$ dimensional set $\Gamma^{\rho}$ ), or, in an equivalent way, as the set of functions $u \in L^{1}(\Omega) \operatorname{such}$ that $\sup \left\{\int_{\Omega} u \operatorname{div} \varphi: \varphi \in C^{1}(\bar{\Omega})\right.$, spt. of $\varphi \subset \Omega \cup \Gamma^{\rho},|\varphi(x)| \leq 1$ for every $\left.x \in \Omega\right\}<+\infty$.
} 
where $E$ is the natural extension of (1) to finite-perimeter partitions:

$$
\begin{aligned}
E(\{S, L, V\}, u)= & \frac{1}{2} \int_{S} A \mathrm{e}(u)(x): \mathrm{e}(u)(x) \mathrm{d} x \\
& -\int_{S} f(x) \cdot u(x) \mathrm{d} x-\int_{\bar{\partial}_{\star} L} p(x) u(x) \cdot \nu_{L}(x) \mathrm{d} \mathcal{H}^{N-1}(x) \\
= & \frac{1}{2} \int_{\Omega} \mathbf{1}_{S}(x) A \mathrm{e}(u)(x): \mathrm{e}(u)(x) \mathrm{d} x \\
& -\int_{\Omega} \mathbf{1}_{S}(x) f(x) \cdot u(x) \mathrm{d} x+\int_{\Omega \cup \Gamma^{\rho}} p(x) u(x) \cdot D \mathbf{1}_{L}(x) .
\end{aligned}
$$

Notice that $C \in[0,+\infty]$ since $E(\{S, L, V\}, 0)=0$. Given $\lambda>0$, our weak problem is now:

$$
\min _{S, L, V} C(S, L, V)+\lambda \bar{\Lambda}(S, L, V)
$$

where the minimum has to be taken among all finite-perimeter partitions $S, L, V$ with given proportion $|S|=$ $\theta_{S}|\Omega|,|L|=\theta_{L}|\Omega|$. We have the following result, valid under the technical hypothesis (H).

Theorem 1.2 (Existence of a solution of the weak problem). Assume there exists a finite-perimeter partition $S$, $L, V$ of finite compliance $C(S, L, V)$. Then problem (9) admits a solution.

This result is simply a consequence of the next approximation theorem.

\subsection{The fictitious material formulation}

In practice, it is very difficult to address the minimization of (9), numerically. The two main reasons to this are that problem (7) generally does not admit a minimizer (for an arbitrary partition $S, L, V$ ), and that we need to represent the free (unknown) boundaries of the sets $S, L$ an $V$ (or the discontinuities of their characteristic functions).

Since we do not know how to make a correct numerical analysis of our problem, we will consider variational approximations of our energies, in the setting of $\Gamma$-convergence. We refer to [5] and [20] for monographs on this notion of convergence, introduced by De Giorgi for the approximation of variational problems. See Appendix A for the main definitions and properties. Our aim is to find approximate energies whose minimizer(s) are close, in some sense, to the solution(s) of (9).

To overcome the first difficulty, we make the classical assumption that the set $L \cup V$ is also filled with a "fictitious material" of very small (in the sense of quadratic forms) elasticity tensor. In practice (but there would be many other ways to implement this idea, we describe the simplest) we fix a small parameter $\delta>0$ and assume that the elasticity tensor is $A$ in $S$ and $\delta A$ in $L \cup V$, so that the energy (8) becomes

$$
\begin{aligned}
E_{\delta}(\{S, L, V\}, u)= & \frac{1}{2} \int_{\Omega}\left(\delta+(1-\delta) \mathbf{1}_{S}(x)\right) A \mathrm{e}(u)(x): \mathrm{e}(u)(x) \mathrm{d} x \\
& -\int_{\Omega} \mathbf{1}_{S}(x) f(x) \cdot u(x) \mathrm{d} x+\int_{\Omega \cup \Gamma^{\rho}} p(x) u(x) \cdot D \mathbf{1}_{L}(x) .
\end{aligned}
$$

Again, the compliance is simply

$$
C_{\delta}(S, L, V)=-2 \min _{u \in X_{u}} E_{\delta}(\{S, L, V\}, u)
$$


(the existence of the minimizer will be discussed in Sect. 3.2 for a general energy) and the approximated problem is

$$
\min _{S, L, V} C_{\delta}(S, L, V)+\lambda \bar{\Lambda}(S, L, V)
$$

where again the minimum is taken among the finite-perimeter partitions $S, L, V$ with given proportion $|S|=$ $\theta_{S}|\Omega|,|L|=\theta_{L}|\Omega|$. The next result again is valid under the technical hypothesis $(\mathrm{H})$.

Theorem 1.3 (Convergence of the solutions of the fictitious material problem). For every $\delta>0$ small, problem (12) admits a solution. Moreover, assuming again that there exists a finite-perimeter partition $S, L, V$ such that $C(S, L, V)<+\infty$, if $S_{\delta}, L_{\delta}, V_{\delta}$ are solutions of (12) for $\delta>0$ small, then there exists a sequence $\left(\delta_{j}\right)_{j \geq 1}, \delta_{j} \downarrow 0$ as $j \rightarrow \infty$, and a finite-perimeter partition $S, L, V$ such that $\mathbf{1}_{S_{\delta_{j}}} \rightarrow \mathbf{1}_{S}, \mathbf{1}_{L_{\delta_{j}}} \rightarrow \mathbf{1}_{L}, \mathbf{1}_{V_{\delta_{j}}} \rightarrow \mathbf{1}_{V}$ in $L^{1}(\Omega)$, and $S, L, V$ is a solution of $(9)$.

Theorem 1.3 will be established in Section 4, as a consequence of Corollary 4.2 and of the analysis in Section 4.3. Notice that it yields Theorem 1.2.

\subsection{A phase-field approximation}

Now, we can compute an approximation of our solution, provided that we are able to solve problem (12) for a small enough $\delta$. However, this problem is not easily solved because the unknown is a finite-perimeter partition. As it is classical, we will represent this partition by means of a $B V$ "density" function taking three values and then, introducing a new scale parameter $\varepsilon$, approximate this density by smoother functions.

We thus introduce a phase field $\rho$ in order to represent the partition of $\Omega$ into the three phases: ideally $\rho$ should be a function taking just the three values $-1,0,1(-1=$ void $V, 0=$ structure $S, 1=\operatorname{liquid} L)$. In the approximate models, however, $\rho$ will be allowed to take any real value.

We introduce a new notation for the subsets $\Gamma_{S}, \Gamma_{L}, \Gamma_{V}$ of $\partial \Omega$ : in what follows they will be denoted respectively by $\Gamma_{0}^{\rho}, \Gamma_{1}^{\rho}, \Gamma_{-1}^{\rho}$, in order to emphasize the fact that they are the parts of the boundary where we want to ensure a Dirichlet condition for the phase $\rho\left(\rho=\alpha\right.$ on $\left.\Gamma_{\alpha}^{\rho}\right)$. We still denote $\Gamma^{\rho}=\cup_{\alpha=-1,0,1} \Gamma_{\alpha}^{\rho}$, and hypothesis $(\mathrm{H})$ states that $\partial \Omega=\Gamma^{u} \cup \Gamma^{\rho}$.

We consider a triple-well (continuous) potential $W(t)$ such that $W(t)>0$ except at $t=-1,0,1$. For instance, we can consider $W(t)=8 t^{2}\left(t^{2}-1\right)^{2}$. For simplicity we assume that $W$ is even and that $\int_{-1}^{0} \sqrt{2 W(t)} \mathrm{d} t=$ $\int_{0}^{1} \sqrt{2 W(t)} \mathrm{d} t=1$, but other (more general) potentials would lead to similar results with a different penalization of the interfaces. We let $X_{\rho}=\left\{\rho \in H^{1}(\Omega): \rho=\alpha\right.$ on $\left.\Gamma_{\alpha}^{\rho}, \alpha=-1,0,1\right\}$ and introduce the energy, for $\rho \in L^{1}(\Omega)$,

$$
\Lambda_{\varepsilon}(\rho)= \begin{cases}\frac{\varepsilon}{2} \int_{\Omega}|\nabla \rho(x)|^{2}+\frac{1}{\varepsilon} \int_{\Omega} W(\rho(x)) \mathrm{d} x & \text { if } \rho \in X_{\rho} \\ +\infty & \text { otherwise. }\end{cases}
$$

This kind of energy was first introduced by Cahn and Hilliard [13] in order to represent the interfacial energy of a mixture of fluids, and then widely studied (see for instance $[1,32,33]$ ).

Next, we introduce the space

$$
\bar{X}_{\rho}=\left\{\rho \in B V\left(\Omega \cup \Gamma^{\rho} ;\{-1,0,1\}\right): \rho^{\operatorname{ext}}(x)=\alpha \text { on } \Gamma_{\alpha}^{\rho}, \alpha=-1,0,1\right\}
$$

where as previously $B V\left(\Omega \cup \Gamma^{\rho} ;\{-1,0,1\}\right)$ denotes the space of functions $\rho$ that belong to $B V(\Omega ;\{-1,0,1\})$ and are considered to have an "exterior trace" $\rho^{\text {ext }}$ on the part $\Gamma^{\rho}$ of $\partial \Omega$, so that their derivative is a measure acting on the continuous functions with compact support in $\Omega \cup \Gamma^{\rho}$, defined by the duality formula (5). 


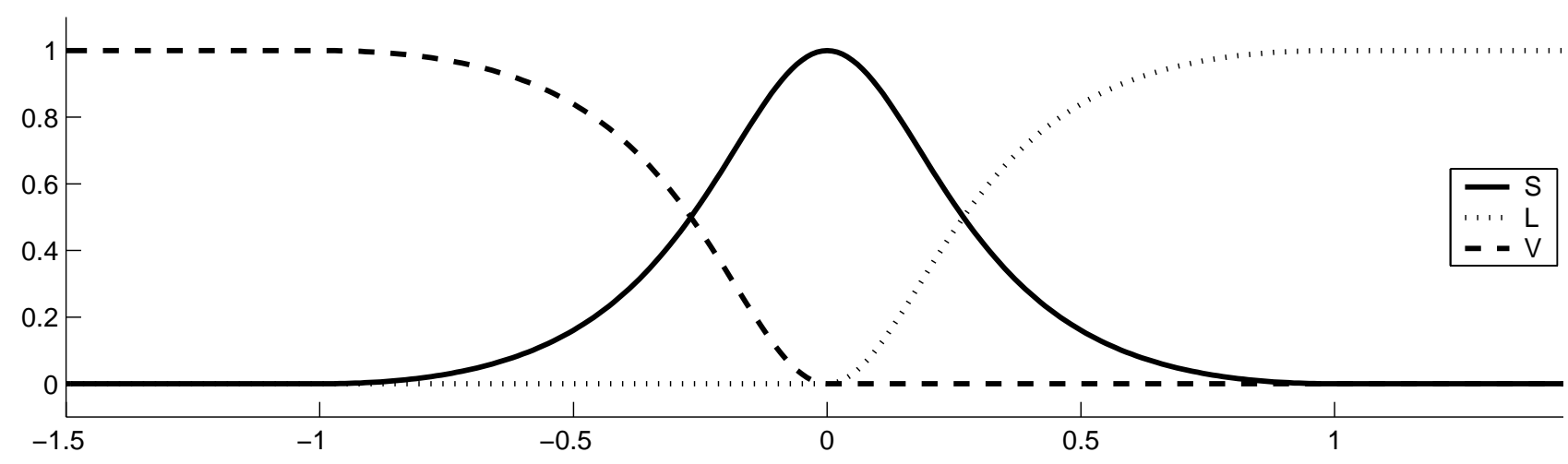

Figure 1. The functions $V, L, S$.

A function $\rho \in \bar{X}_{\rho}$ has therefore a derivative which is the measure in $\mathcal{M}\left(\Omega \cup \Gamma^{\rho} ; \mathbb{R}^{N}\right)$ given by

$$
D \rho=D \rho\left\llcorner\Omega+\sum_{\alpha=-1,0,1}\left(\alpha-\rho^{\text {int }}(x)\right) \nu_{\Omega}(x) \mathcal{H}^{N-1}\left\llcorner\Gamma_{\alpha}^{\rho},\right.\right.
$$

were as before $\rho^{\text {int }}$ denotes the interior trace of $\rho$ on $\partial \Omega$.

This is the correct, weak way of enforcing the condition " $\rho=\alpha$ on $\Gamma_{\alpha}^{\rho}$ " for functions $\rho$ that have only $B V$ regularity. In this setting, the total variation of $\rho$ on $\Omega \cup \Gamma^{\rho}$ includes a penalization if the inner trace $\rho^{\text {int }}$ disagrees with the Dirichlet condition:

$$
|D \rho|\left(\Omega \cup \Gamma^{\rho}\right)=|D \rho|(\Omega)+\sum_{\alpha=-1,0,1} \int_{\Gamma_{\alpha}^{\rho}}\left|\rho^{\text {int }}(x)-\alpha\right| \mathrm{d} \mathcal{H}^{N-1}(x) .
$$

Recalling definition (6), we see that

$$
|D \rho|\left(\Omega \cup \Gamma^{\rho}\right)=\bar{\Lambda}(\{\rho=0\},\{\rho=1\},\{\rho=-1\}) .
$$

For this reason, we will still denote by $\bar{\Lambda}$ the functional of $L^{1}(\Omega)$

$$
\bar{\Lambda}: \rho \mapsto \begin{cases}|D \rho|\left(\Omega \cup \Gamma^{\rho}\right) & \text { if } \rho \in \bar{X}_{\rho}, \\ +\infty & \text { otherwise. }\end{cases}
$$

Then, we introduce three "phase indicators" that we denote by $S, L, V: \mathbb{R} \rightarrow(0,1)$. These are smooth functions such that $S(0)=1, S(t)=0$ if $|t| \geq 1, S$ increases on $(-1,0)$ and decreases on $(0,1) ; L(t)=0$ if $t \leq 0, L(t)=1$ if $t \geq 1$, and $L$ increases on [0,1]; and $V=1-L-S$ (see Fig. 1 for a possible choice of the phase indicators). For every $\rho \in L^{1}(\Omega)$, we will interpret $V(\rho(x)), L(\rho(x)), S(\rho(x))$ as the proportion of each "phase" (void, liquid and structure) at the point $x$. If $\rho \in \bar{X}_{\rho}$, then clearly $V(\rho)=\rho^{-}=\mathbf{1}_{\{\rho=-1\}}, L(\rho)=\rho^{+}=\mathbf{1}_{\{\rho=1\}}$, and $S(\rho)=\mathbf{1}_{\{\rho=0\}}$. In this case for simplicity $V(\rho)$ (respectively, $L(\rho), S(\rho)$ ) will also denote the finite-perimeter set where $V(\rho(x))=1$ (resp., $L(\rho(x))=1, S(\rho(x))=1$ ), as in the previous sections.

Given $\rho \in \bar{X}_{\rho}$ we also have that $L(\rho) \in B V\left(\Omega \cup \Gamma^{\rho} ;\{-1,0,1\}\right)$ with an exterior trace $L(\rho)^{\text {ext }}=L\left(\rho^{\text {ext }}\right)=1$ on $\Gamma_{1}^{\rho}=\Gamma_{L}$ and 0 on $\Gamma_{-1}^{\rho} \cup \Gamma_{0}^{\rho}=\Gamma_{V} \cup \Gamma_{S}$, and the derivative of $L(\rho)$ is defined as in (5):

$$
\int_{\Omega \cup \Gamma^{\rho}} \varphi(x) \cdot D L(\rho)=\int_{\Gamma_{1}^{\rho}} \varphi(x) \cdot \nu_{\Omega}(x) \mathrm{d} \mathcal{H}^{N-1}(x)-\int_{L(\rho)} \operatorname{div} \varphi(x) \mathrm{d} x,
$$


and supported again by the extended measure-theoretical boundary of $L(\rho), \bar{\partial}_{\star} L(\rho)=\partial_{\star} L(\rho) \cup\left\{x \in \Gamma^{\rho}\right.$ : $\left.L\left(\rho^{\mathrm{ext}}\right) \neq L\left(\rho^{\mathrm{int}}\right)\right\}$.

For $\delta>0, \rho \in \bar{X}_{\rho}, u \in X_{u}$, the linear elasticity energy (10) becomes in our new notations

$$
\begin{aligned}
E_{\delta}(\rho, u)= & \frac{1}{2} \int_{\Omega}(\delta+(1-\delta) S(\rho(x))) A \mathrm{e}(u)(x): \mathrm{e}(u)(x) \mathrm{d} x \\
& -\int_{\Omega} S(\rho(x)) f(x) \cdot u(x) \mathrm{d} x+\int_{\Omega \cup \Gamma^{\rho}} p(x) u(x) \cdot D L(\rho) .
\end{aligned}
$$

On the other hand, if $\rho \in X_{\rho}$, we let

$$
\begin{aligned}
E_{\delta}(\rho, u)= & \frac{1}{2} \int_{\Omega}(\delta+(1-\delta) S(\rho(x))) A \mathrm{e}(u)(x): \mathrm{e}(u)(x) \mathrm{d} x \\
& -\int_{\Omega} S(\rho(x)) f(x) \cdot u(x)+\int_{\Omega} p(x) u(x) \cdot \nabla L(\rho)(x) \mathrm{d} x .
\end{aligned}
$$

Then, integrating by part the last term, and recalling hypothesis $(\mathrm{H})$ that implies that $u \cdot \nu_{\Omega}=0$ out of $\Gamma^{\rho}$, we see that both formulae (15) and (16) may be written

$$
\begin{aligned}
E_{\delta}(\rho, u)= & \frac{1}{2} \int_{\Omega}(\delta+(1-\delta) S(\rho(x))) A \mathrm{e}(u)(x): \mathrm{e}(u)(x) \mathrm{d} x \\
& -\int_{\Omega} S(\rho(x)) f(x) \cdot u(x) \mathrm{d} x \\
& -\int_{\Omega} L(\rho(x)) \operatorname{div}(p u)(x) \mathrm{d} x+\int_{\Gamma_{1}^{\rho}} p(x) u(x) \cdot \nu_{\Omega}(x) \mathrm{d} \mathcal{H}^{N-1}(x),
\end{aligned}
$$

for every $u \in X_{u}$ and $\rho \in X_{\rho} \cup \bar{X}_{\rho}$. The last expression is in fact well defined for every $\rho \in L^{1}(\Omega)$.

Remark 1.4. We see that equation (17) holds if we just assume that on $\partial \Omega \backslash\left(\Gamma^{u} \cup \Gamma_{1}^{\rho}\right), \rho \leq 0$, so that all our results still hold true if we assume a slightly more complicated Dirichlet boundary condition on this part of the boundary than $\rho=-1$ on $\Gamma_{-1}^{\rho}$ and $\rho=0$ on $\Gamma_{0}^{\rho}$.

We can extend the definition (11) of the compliance to any $\rho \in L^{1}(\Omega)$, simply letting

$$
C_{\delta}(\rho)=-2 \inf _{u \in X_{u}} E_{\delta}(\rho, u) .
$$

Problem (12) admits the equivalent formulation

$$
\min _{\rho \in L^{1}(\Omega)} C_{\delta}(\rho)+\lambda \bar{\Lambda}(\rho)
$$

where the minimum must be taken among the densities $\rho$ such that $\int_{\Omega} S(\rho(x)) \mathrm{d} x=\theta_{S}|\Omega|, \int_{\Omega} L(\rho(x)) \mathrm{d} x=$ $\theta_{L}|\Omega|$.

For $\varepsilon>0$ we have a new approximated problem

$$
\min _{\rho \in L^{1}(\Omega)} C_{\delta}(\rho)+\lambda \Lambda_{\varepsilon}(\rho),
$$

under the same constraints. Then, we have the following theorem, on which our numerical implementation is based: 
Theorem 1.5 (Convergence of the solutions of the phase-field problem). Assume $\delta>0$ is fixed. Then, for every $\varepsilon>0$ small, problem (20) admits a solution. Moreover, if $\rho_{\varepsilon}$ are solutions of (20) for $\varepsilon>0$ small, then there exists a sequence $\left(\varepsilon_{j}\right)_{j \geq 1}, \varepsilon_{j} \downarrow 0$ as $j \rightarrow \infty$, and $\rho \in \bar{X}_{\rho}$ such that $\rho_{\varepsilon_{j}} \rightarrow \rho$ in $L^{1}$ and $\rho$ is a solution of (19) (equivalently $(S(\rho), L(\rho), V(\rho))=(\{\rho=0\},\{\rho=1\},\{\rho=-1\})$ is a solution of (12)).

The proof of this theorem is given in Section 3. It is a consequence of the Corollary 3.4.

\section{THE NUMERICAL IMPLEMENTATION}

In this section, we present and detail the actual numerical implementation of our problem, which relies on the approximation results discussed in the previous sections.

The actual implementation described in this section has been made only in the bidimensional case $(N=2)$, however, the techniques described here would also apply to the case $N=3$ without changes.

The base of our numerical algorithm is to be found in Theorem 1.5, which states that the minimizers of the "fictitious material" problem (19) approximate that of the original one (12). Of course, in view of a numerical implementation, one cannot really send the regularization parameters $\delta$ and $\varepsilon$ to 0 but one can set these parameters to an arbitrary small value and numerically compute a minimizer for $C_{\delta}+\lambda \Lambda_{\varepsilon}$.

This approach is in spirit very similar to the so-called diffuse interface methods widely used in materials science. Indeed, our numerical algorithm uses several ideas from the literature (see for instance $[16,23,31]$ ). In that framework, our original problem (9) would be the "sharp interface" problem and its approximation would be referred to as the "diffuse interface" problem, which is to be numerically implemented.

Remark 2.1. In our model, the surface energy $\Lambda_{\varepsilon}$ is used only to prevent from fast oscillations, and then from homogenization, of the design variable, as it penalizes small scale patterns (this is illustrated in Sect. 2.3.2). Therefore, the parameter $\lambda$ has to be set so that the leading term in the objective function is the compliance.

Unfortunately, the numerical implementation of the regularized surface energy is also the most challenging part. This is why most of the techniques described in this section aim at its efficient implementation.

In order to deal with the volume constraints on the phases, we introduce three Lagrange multipliers which we update at each iteration. This is discussed at the beginning of the next section.

Then, the main difficulty related to the compliance part of the objective function $C_{\delta}$ is that it does not depend explicitly on the phase field $\rho$ but rather on the equilibrium displacement $u_{\rho}$ associated with this design. However, the sensitivity of the compliance with respect to a design change can be computed in closed form, as it is shown in Lemma 2.2, and one can implement a gradient-based optimization algorithm.

The regularized surface energy is non-convex which means that a descent algorithm might be unstable or converge to a local minimizer. In our implementation, the former issue is addressed by the use of a semi-implicit descent scheme and a continuation method reduces the effects of the latter. Both techniques are detailed in Section 2.1.

Lastly, the actual implementation by means of piecewise linear finite elements is detailed in Section 2.2 , and some numerical experiments are shown in Section 2.3

\subsection{Algorithm}

A first problem in the implementation of the numerical minimization of $C_{\delta}+\lambda \Lambda_{\varepsilon}$ is to deal with the set of volume fraction constraints.

$$
\left\{\begin{array}{l}
\int_{\Omega} S(\rho) \mathrm{d} x=|\Omega| \theta_{S} \\
\int_{\Omega} L(\rho) \mathrm{d} x=|\Omega| \theta_{L} \\
\int_{\Omega} V(\rho) \mathrm{d} x=|\Omega| \theta_{V}
\end{array}\right.
$$

where $\theta_{S}+\theta_{L}+\theta_{V}=1$. 
As these constraints depend nonlinearly on the phase field, their implementation is complicated. In the proof of Theorem 1.5, this difficulty is circumvented by introducing a penalized surface energy which takes value $+\infty$ whenever the constraints are not satisfied. Of course, for practical purposes, we cannot use this formulation; instead, we introduce three non-negative Lagrange multipliers, $\lambda_{S}, \lambda_{L}$, and $\lambda_{V}$ and the Lagrangian

$$
\begin{aligned}
\mathcal{L}\left(\rho,\left(\lambda_{S}, \lambda_{L}, \lambda_{V}\right)\right)= & C_{\delta}(\rho)+\lambda \Lambda_{\varepsilon}(\rho)+\lambda_{S} \int_{\Omega}\left(\widetilde{S}(\rho)-\theta_{S}\right) \mathrm{d} x \\
& +\lambda_{L} \int_{\Omega}\left(\widetilde{L}(\rho)-\theta_{L}\right) \mathrm{d} x+\lambda_{V} \int_{\Omega}\left(\widetilde{V}(\rho)-\theta_{V}\right) \mathrm{d} x .
\end{aligned}
$$

Here, the functions $\widetilde{V}, \widetilde{S}$, and $\widetilde{L}$ are three interpolation functions which coincide with $V$, $S$, and $L$ at the points $-1,0$, and 1 and such that $\widetilde{S}+\widetilde{L}+\widetilde{V}=1$. The purpose of such a substitution might sounds somewhat unclear at this point but its soundness, in the numerical implementation will be justified further in the paper. Its goal is detailed in Section 2.3, while Remark 3.2 reminds that this substitution has no effect on the theoretical results.

Unfortunately, the numerical choice of the Lagrange multipliers associated with given volume fractions is not obvious. Actually, their existence for a given set of volume fractions is not even granted as we do not have any information on the continuity of the solution of the primal problem (the design), with respect to a small change of the dual variables.

However, in the practical implementation, we assume that the Lagrange multipliers exist. In this case, it is easy to see that they are defined up to the addition of a constant so that one can assume their positiveness without loss of generality. Another way to see it is to notice that the equalities in (21) could be replaced by three inequalities of the same type, in which case the multipliers would be required to be of the same sign.

Then, the goal of our optimization algorithm is to find a set $\rho, \lambda_{S}, \lambda_{L}, \lambda_{V}$ satisfying the Kuhn and Tucker conditions associated with the Lagrangian $\mathcal{L}$.

For that matter we use an iterative scheme: at each step, we first update the density field based on the sensitivity of the objective function with respect to a design change. Then, we modify the Lagrange multipliers according to the following rule:

$$
\left\{\begin{array}{l}
\lambda_{S}^{n}=\frac{\lambda_{S}^{n-1}}{|\Omega|} \int_{\Omega} \frac{\widetilde{S}\left(\rho^{n}\right)}{\theta_{S}} \mathrm{~d} x \\
\lambda_{L}^{n}=\frac{\lambda_{L}^{n-1}}{|\Omega|} \int_{\Omega} \frac{\widetilde{L}\left(\rho^{n}\right)}{\theta_{L}} \mathrm{~d} x \\
\lambda_{V}^{n}=\frac{\lambda_{V}^{n-1}}{|\Omega|} \int_{\Omega} \frac{\widetilde{V}\left(\rho^{n}\right)}{\theta_{V}} \mathrm{~d} x .
\end{array}\right.
$$

The update of the phase field $\rho$ is more complicated since it requires the computation of the sensitivity of the objective function. The derivative of the surface energy or the constraints with respect to $\rho$ is straightforward but that of the compliance is less simple. This is because $C_{\delta}$ does not depend explicitly on $\rho$ but rather through the work of the forces for the equilibrium displacement, linked to $\rho$ through equation (11). However, this derivative can be computed in a closed form, as it is stated in the following lemma:

Lemma 2.2. Let $\rho \in L^{1}(\Omega)$ and $\psi \in L^{\infty}(\Omega)$. Then the derivative of the compliance with respect to a design change in the direction $\psi$ is given by

$$
\lim _{t \rightarrow 0} \frac{C_{\delta}(\rho+t \psi)-C_{\delta}(\rho)}{t}-2 \int_{\Omega}\left(\frac{1-\delta}{2} S^{\prime}(\rho) A \mathrm{e}\left(u_{\rho}\right): \mathrm{e}\left(u_{\rho}\right)-S^{\prime}(\rho) f \cdot u_{\rho}-L^{\prime}(\rho) \operatorname{div}\left(p u_{\rho}\right)\right) \psi \mathrm{d} x
$$

where $u_{\rho}$ is the minimizer of $E_{\delta}(\rho, u)$ over $u \in X_{u}$. 
Proof. For every $t$ near zero let $\rho_{t}=\rho+t \psi$ and $u_{t}$ be the minimizer of $E_{\delta}\left(\rho_{t}, u\right)$ over $u \in X_{u}$. Taking the difference of $(33)$ for $\left(\rho_{t}, u_{t}\right)$ and for $\left(\rho_{0}, u_{0}\right)=\left(\rho, u_{\rho}\right)$ and dividing by $t$ gives

$$
\begin{gathered}
\int_{\Omega}(1-\delta) \frac{S\left(\rho_{t}\right)-S(\rho)}{t} A \mathrm{e}\left(u_{t}\right): \mathrm{e}(v) \mathrm{d} x+\int_{\Omega}(\delta+(1-\delta) S(\rho)) A \mathrm{e}\left(\frac{u_{t}-u_{0}}{t}\right): \mathrm{e}(v) \mathrm{d} x \\
=\int_{\Omega} \frac{S\left(\rho_{t}\right)-S(\rho)}{t} f \cdot v \mathrm{~d} x+\int_{\Omega} \frac{L\left(\rho_{t}\right)-L(\rho)}{t} \operatorname{div}(p v) \mathrm{d} x
\end{gathered}
$$

for every $v \in X_{u}$. Let $w_{t}=\left(u_{t}-u_{0}\right) / t \in X_{u}$. Notice that $\left(S\left(\rho_{t}\right)-S(\rho)\right) / t \leq\left\|S^{\prime}\right\|_{\infty}|\psi|$ and $\left(L\left(\rho_{t}\right)-L(\rho)\right) / t$ $\leq\left\|L^{\prime}\right\|_{\infty}|\psi|$ are bounded in $L^{\infty}(\Omega)$. Therefore, taking a sup over $v \in X_{u},\|\mathrm{e}(v)\|_{L^{2}} \leq 1$ in (25) and using Korn's inequality $(31)$ we get that $\left(w_{t}\right)$ is uniformly bounded in $H^{1}\left(\Omega ; \mathbb{R}^{N}\right)$. Let $w_{0}$ be the weak $H^{1}$-limit of some sequence $\left(w_{t_{k}}\right)_{k \geq 1}$. Passing to the limit in $(25)$, and noticing that $\left(S\left(\rho_{t}\right)-S(\rho)\right) / t \rightarrow S^{\prime}(\rho) \psi$ and $\left(L\left(\rho_{t}\right)-L(\rho)\right) / t \rightarrow L^{\prime}(\rho) \psi$ a.e. in $\Omega$, we get that $w_{0} \in X_{u}$ is characterized by

$$
\begin{gathered}
\int_{\Omega}(1-\delta) S^{\prime}(\rho) A \mathrm{e}\left(u_{0}\right): \mathrm{e}(v) \psi \mathrm{d} x+\int_{\Omega}(\delta+(1-\delta) S(\rho)) A \mathrm{e}\left(w_{0}\right): \mathrm{e}(v) \mathrm{d} x \\
=\int_{\Omega} S^{\prime}(\rho) f \cdot v \psi \mathrm{d} x+\int_{\Omega} L^{\prime}(\rho) \operatorname{div}(p v) \psi \mathrm{d} x
\end{gathered}
$$

for every $v \in X_{u}$. In particular $w_{0}$ is unique and we get that $\lim _{t \rightarrow 0} w_{t}=w_{0}$ weakly in $H^{1}$.

Since

$$
C_{\delta}\left(\rho_{t}\right)=\int_{\Omega}\left(\delta+(1-\delta) S\left(\rho_{t}\right)\right) A \mathrm{e}\left(u_{t}\right): \mathrm{e}\left(u_{t}\right) \mathrm{d} x
$$

we have

$$
\frac{C_{\delta}\left(\rho_{t}\right)-C_{\delta}(\rho)}{t}=\int_{\Omega}(1-\delta) \frac{S\left(\rho_{t}\right)-S(\rho)}{t} A \mathrm{e}\left(u_{t}\right): \mathrm{e}\left(u_{t}\right)+(\delta+(1-\delta) S(\rho)) A \mathrm{e}\left(w_{t}\right): \mathrm{e}\left(u_{t}+u_{0}\right) \mathrm{d} x
$$

Notice that $u_{t}-u_{0}=t w_{t}$ so that $u_{t}$ goes to $u_{0}$ strongly in $H^{1}(\Omega)$, and also that

$$
\frac{S\left(\rho_{t}\right)-S(\rho)}{t} \mathrm{e}\left(u_{t}\right)=\frac{S\left(\rho_{t}\right)-S(\rho)}{t} \mathrm{e}\left(u_{0}\right)+\left(S\left(\rho_{t}\right)-S(\rho)\right) \mathrm{e}\left(w_{t}\right)
$$

and since $\left|\left(S\left(\rho_{t}\right)-S(\rho)\right) \mathrm{e}\left(w_{t}\right)\right| \leq|t|\left\|S^{\prime}\right\|_{\infty}|\psi|\left|\mathrm{e}\left(w_{t}\right)\right|$ a.e. in $\Omega$ we deduce that as $t$ goes to zero, e $\left(u_{t}\right)\left(S\left(\rho_{t}\right)\right.$ $-S(\rho)) / t$ goes strongly to $S^{\prime}(\rho) \psi$ e $\left(u_{0}\right)$ in $L^{2}$. Hence, we get by passing to the limit in $(27)$

$$
\lim _{t \rightarrow 0} \frac{C_{\delta}\left(\rho_{t}\right)-C_{\delta}(\rho)}{t}=\int_{\Omega}(1-\delta) S^{\prime}(\rho) A \mathrm{e}\left(u_{0}\right): \mathrm{e}\left(u_{0}\right) \psi+2(\delta+(1-\delta) S(\rho)) A \mathrm{e}\left(w_{0}\right): \mathrm{e}\left(u_{0}\right) \mathrm{d} x .
$$

We deduce (24) from (26) with $v=u_{0}=u_{\rho}$ and (28).

Remark 2.3. Equation (24) can be rewritten as

$$
\lim _{t \rightarrow 0} \frac{C_{\delta}(\rho+t \psi)-C_{\delta}(\rho)}{t}=-\left.2 \lim _{t \rightarrow 0} \frac{E_{\delta}(\rho+t \psi, u)-E_{\delta}(\rho, u)}{t}\right|_{u=u_{\rho}},
$$

which is a classical identity in compliance optimization. 
The easiest way to update the density field would be through an explicit steepest descent scheme. In that case, the $n$-th descent iteration, would then write as

$\rho^{n}=\rho^{n-1}-r\left\{D_{\rho} C_{\delta}\left(\rho^{n-1}\right)+\frac{\lambda}{\varepsilon} W^{\prime}\left(\rho^{n-1}\right)-2 \lambda \varepsilon \Delta \rho^{n-1}+\lambda_{S} \widetilde{S}^{\prime}\left(\rho^{n-1}\right)+\lambda_{L} \widetilde{L}^{\prime}\left(\rho^{n-1}\right)+\lambda_{V} \widetilde{V}^{\prime}\left(\rho^{n-1}\right)\right\}$

where $D_{\rho} C_{\delta}(\rho)$ is the derivative of the compliance given by the previous lemma and $r$ is the descent step. Unfortunately, such a scheme is very unstable and requires a very small descent step. It is easy to see, for instance, that due to the Laplacian term, a fully explicit scheme is unstable as soon as $r \geq c \lambda \varepsilon h^{2}$ for some $c>0$, where $h$ is the discretization characteristic length. It is actually known that the actual upper bound on the step for the surface term is of the order of $\lambda \varepsilon h^{4}$, which implies a very poor convergence rate. Considering that the evaluation of $D_{\rho} C_{\delta}(\rho)$ requires the computation of the equilibrium displacement associated with the design $\rho$, and hence the solving of an elasticity problem, this is not acceptable.

In order to enhance the convergence rate and following [35,37] and somehow [16], we rewrite the non-convex part $W$ of the surface energy as $W(x)+k \frac{x^{2}}{2}-k \frac{x^{2}}{2}$, where $k \leq-\min _{(-1,1)} W^{\prime \prime}$ so that $F(x)=W(x)+k \frac{x^{2}}{2}$ is convex in $(-1,1)$. Then, it is possible to speed up the numerical scheme by using an implicit scheme for the Laplacian and quadratic parts of the objective function and an explicit scheme for the remaining terms. Eventually, the iteration of the semi-implicit scheme we use writes as

$$
\begin{aligned}
\left(1-r k \frac{\lambda}{\varepsilon}-2 r \lambda \varepsilon \Delta\right) \rho^{n}= & \rho^{n-1}-r\left\{D_{\rho} C_{\delta}\left(\rho^{n-1}\right)+\frac{\lambda}{\varepsilon} F^{\prime}\left(\rho^{n-1}\right)\right. \\
& \left.+\lambda_{S} \widetilde{S}^{\prime}\left(\rho^{n-1}\right)+\lambda_{L} \widetilde{L}^{\prime}\left(\rho^{n-1}\right)+\lambda_{V} \widetilde{V}^{\prime}\left(\rho^{n-1}\right)\right\}
\end{aligned}
$$

The main difference between our semi-implicit scheme and those used in the above-mentioned articles is that we deal with the Laplacian implicitly while they implement it explicitly. This is because our implementation choice is to use unstructured finite elements instead of finite difference (see Sect. 2.2 for a discussion of the reasons leading to this choice). Hence, evaluating the Laplacian of the density field requires the computation of its projection onto the considered finite element space, i.e. solving a heat equation, which in terms of computational cost is equivalent to that of solving the Laplace equation associated with the iteration formula (29).

Remark 2.4. It is clear that if the scheme converges, the resulting fields $\rho, \lambda_{S}, \lambda_{L}$, and $\lambda_{V}$ satisfy the KarushKuhn and Tucker conditions for the Lagrangian $\mathcal{L}$. However, proving the convergence of the scheme is an open problem.

The last ingredient in our numerical implementations is linked to the problem of local minimizers. As we are applying a gradient algorithm to a non-convex problem, our scheme might converge only to a local minimizer. This is a very common problem and several methods are to be found in the literature. In [27], for instance, it is suggested to use an iterative algorithm and gradually increase the perimeter penalization factor, denoted $\lambda$ in our case. The underlying idea is to let the algorithm explore as many designs as possible and later restrict the exploration space by increasing the penalty factor on the non-convex term. Our solution is related to that idea but is slightly more complicated as it involves both an adaptive step strategy and a continuation method.

Instead of increasing the parameter $\lambda$ with the iteration, we slowly decrease the scale parameter, $\varepsilon$, until it reaches some lower bound. This strategy has two advantages on the previous one. First, we favor the convex terms in the energy during the first iterations, which is the base of a continuation method. Then, it is known that the "front propagation" speed (i.e. the speed at which the edges of the structure can move during the iterative process) decreases with $\varepsilon$. By starting with a "relatively large" value for $\varepsilon$, we let the structure evolve quickly during the first iterations and the design to remain "smooth". Then, when $\varepsilon$ gradually decreases, the field $\rho$ concentrates its values around $-1,0$ and 1 and the design tends to evolve more slowly.

At the same time, we use an adaptive step strategy for the choice of the parameter $r$ in (29). Computing the optimal $r$ (i.e. doing a line search) is feasible but tends to be numerically inefficient as each evaluation of 
our objective function is very expensive. Instead, we gradually increase $r$ with the iterations. Then, we check regularly if the energy of the sequence of designs is decreasing. If not, we go back to the last saved iteration, reduce the step and start again. Remark that if $r . \varepsilon$ stays constant, this scheme results in fixing the weight on the convex part of our objective function while gradually increasing that on the non-convex terms.

\subsection{Implementation}

Again, Remark 2.1 is the base of the main technical choices. As the surface energy is only a perturbation of the compliance, the accuracy of its approximation is not nearly as important as its isotropy. Indeed, the phase field $\rho$ is also used to keep track of the normal direction of the interfaces and hence of the pressure force. If the approximation of the perimeter energy favors some orientations of the boundary of the structure, the pressure force will also tend to match these orientations. In such a case, the perimeter penalization will not only act as a way to prevent from fast oscillation and to enforce a lower bound the scale of the smallest patterns but will also influence the general design.

Of course, as we are doing a two step approximation ( $\Gamma$-convergence first and discretization then), most of the anisotropy induced by a structured discretization described in $[14,34]$ should disappear as the discretization parameter tends to zero. However, there are some numerical evidences that for a fixed mesh size, these effects are noticeable.

This is the reason why we chose to approximate the $\rho$ field by means of piecewise linear finite elements on an unstructured triangular mesh. In this setting the dependency of the design upon the discretization is very easy to check experimentally by running several computations on different meshes. Also, since the leading term is to be the compliance, we do not attempt to obtain a very high precision on the interpolated surface energy and we do not need a very fine mesh or an adaptive meshing technique. Thus, we can use the same discretization for both $\rho$ and $u$, which means that we do not have to compute a projection from one discrete space to another at each iteration.

Our choice of finite elements and non-structured triangulation differs from the classical choice of using finite differences, or finite elements over a structured mesh, as it is often seen in topology optimization, or in the "diffuse interface" methods in material science. It makes the implementation more complicated, but it is also very efficient, in preventing from most of usual numerical issues like mesh dependency, checkerboards, and anisotropy.

Remark 2.5. The approximation of the compliance (18), given itself by the elastic potential (16), and of the surface energy (13) by piecewise linear finite elements does not present any major difficulty, and is not detailed here.

\subsection{Numerical experiments}

Before running any computation, we have to choose the triple-well function $W$ and the interpolation laws $S$, $L$ and $V$. Indeed, while the convergence theorem does not depend on their actual expression, their proper choice can improve the efficiency of the numerical algorithm.

In the numerical experiments, the triple-well function $W$ is given by

$$
W(x)=\alpha \frac{\left(1-x^{2}\right)^{2} x^{2}}{1+2 x^{2}},
$$

where $\alpha$ is such that $\int_{0}^{1} \sqrt{2 W(x)} \mathrm{d} x=1$, i.e. $\alpha \simeq 12.58$. The parameter $k$ in equation (29) is set equal to $\alpha$.

We also choose to use different laws for the volume constraint and the stiffness interpolation function associated with the solid and void phases. Indeed, it is known (see [9] for instance) that if one uses the same interpolation scheme for the volume constraint and the stiffness of a material, one artificially favors the intermediate densities. In our case, it would be a problem since the approximation scheme relies on the density field $\rho$ focusing around the values $-1,0$ and 1 , when $\varepsilon$ goes to 0 . A proper choice for $S$ and $\widetilde{S}$ is such that the 


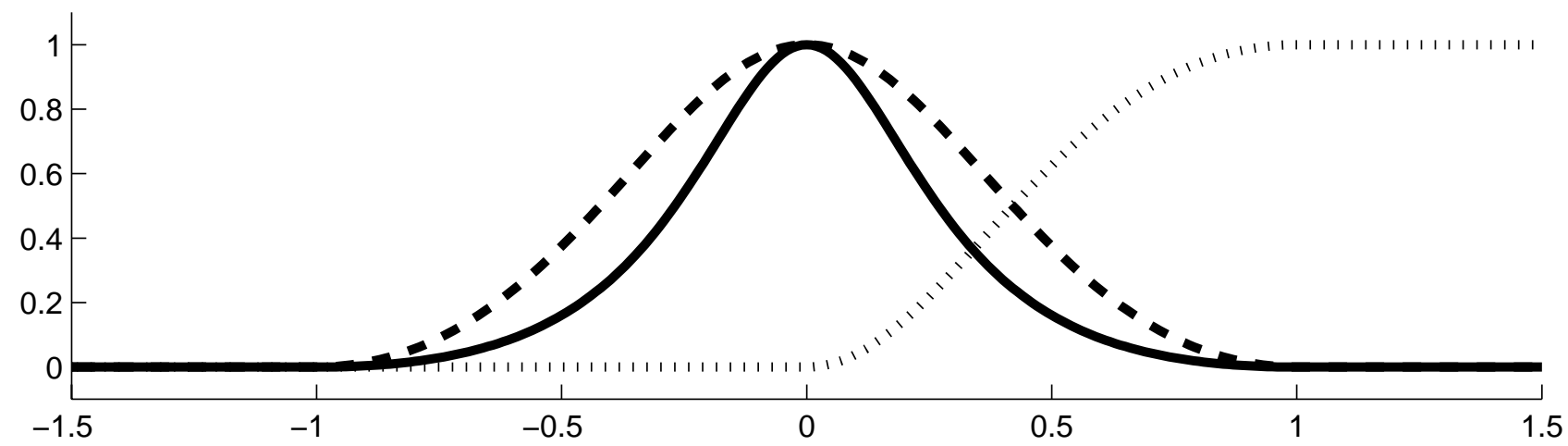

FIgURE 2. The functions $S$ (plain), $\widetilde{S}$ (dashed) and $L$ (dotted) used in the numerical implementation.

intermediate values of the density tend to disappear naturally. Then, one can obtain well "focussed" numerical results for smaller values of the weight on $W$ in the surface energy, i.e. for "larger" $\varepsilon$. This, in turn, permits to use larger descent steps and increase the convergence speed, according to the previous comments ${ }^{4}$.

The interpolation functions used in the implementation are those pictured in Figure 2, namely:

$$
\begin{array}{ll}
S(x)= \begin{cases}0 & \text { if }|x| \geq 1, \\
\frac{\left(1-x^{2}\right)^{2}}{1+10 x^{2}} & \text { otherwise, }\end{cases} & \widetilde{S}(x)= \begin{cases}0 & \text { if }|x| \geq 1, \\
\frac{\left(1-x^{2}\right)^{2}}{1+2 x^{2}} & \text { otherwise, }\end{cases} \\
L(x)= \begin{cases}0 & \text { if } x \leq 0 \\
1-\frac{\left(1-x^{2}\right)^{2}}{1+2 x^{2}} & \text { if } 0 \leq x \leq 1, \\
1 & \text { otherwise, }\end{cases} & \widetilde{L}(x)=L(x) .
\end{array}
$$

In all the numerical experiments presented later, the elastic properties of the material have been chosen such that the Young's modulus $\mathrm{E}$ is equal to 1 and the Poisson's ratio $\nu=.3$ (i.e. $\kappa \simeq .7143$ and $\mu \simeq .3846$ ). The pressure field $p$ also had to be normalized accordingly.

Remark 2.6. In the example we chose to present, we enforce only a constraint on the volume fraction of the solid phase. This is achieved by setting $\lambda_{L}^{0}=\lambda_{V}^{0}=0$ in (23). In that case, one can not rigorously assume the positiveness of the third Lagrange multiplier. This would be true only if one could prove that the compliance of the optimal design is an increasing function of its volume fraction. Unfortunately, such a result is not known in our case, even if it seems very likely, except for some degenerate cases.

\subsubsection{Design of a dam}

The first experiments presented here mimics the design of a dam in a square domain $(0,1) \times(-1,0)$. The pressure field varies linearly with the deepness: $p(x, y)=-y / 2$ and the boundary conditions are the following: on the left edge, $\rho$ is set to be equal to 1 (i.e. a liquid phase is prescribed) and the displacement is left unconstrained. On the right edge, one sets $\rho=-1$ (the void) and $u$ is left free. On the bottom edge, one enforces a Dirichlet boundary condition of both components of the displacement field and the phase is left free. Lastly, the vertical displacement is prescribed on the upper edge and the phase is left free. Using the notations of Section 1, we have that $\Gamma^{u}=(0,1) \times\{-1,0\}, \Gamma_{L}=\{0\} \times(-1,0), \Gamma_{V}=\{1\} \times(-1,0)$ and $\Gamma_{S}=\emptyset$. A schematic representation of the problem is shown in Figure 3 a.

\footnotetext{
${ }^{4}$ One of the referees noted that substituting $\widetilde{S}$ for $S$ as an interpolation function is essentially equivalent to replacing the triple-well potential $W$ with $\widetilde{W}=W+\varepsilon \frac{\lambda_{S}}{\lambda}(\widetilde{S}-S)$ in the surface energy $\Lambda_{\varepsilon}$.
} 
The volume fraction of the material is set to $12 \%$. The discretization, shown in Figure $3 \mathrm{~b}$ consists of 3732 triangular elements and 1944 nodes. In order to prevent from mesh-induced anisotropy, it has no favored direction and is built using the Delaunay-Voronoï mesh generator emc2, developed at the INRIA ${ }^{5}$. The continuation algorithm described earlier is used: the approximation parameter $\varepsilon$ goes from .64 to .32 while the step $r$ varies from $10^{-3}$ to $5 \times 10^{-2}$. The penalization factor for the surface energy has been experimentally set so that at convergence, the compliance is at least 5 times as large as the perimeter energy. Its numerical value is $4 \times 10^{-3}$. We ran the computation until the absolute change on the density, $\left|\rho^{n+1}-\rho^{n}\right|$ is lower than $1.5 \times 10^{-4}$, which took about 1500 iterations. The compliance of the computed design is equal to .191 and the surface energy $3.8 \times 10^{-2}$.

Figure $3 \mathrm{c}$ represents the final design, i.e. the phase field $\rho$ after convergence of the algorithm. The value -1 (associated with the void) is color-coded in white, the value 0 (the solid) in grey and the black corresponds to the value 1 (the liquid). Of course, as we solved the approximated problem for a fixed $\varepsilon$, the function $\rho$ is "smooth". However, its values are mostly concentrated round the three well of the function $W$. The black and the white lines plotted over the density field represent respectively the level-line $1 / 3$ and $-1 / 3$ of the density. They suggest how the design might look like when the relaxation parameter goes to 0 .

The numerical result has a few interesting properties: first, the cavity in the dam is filled with liquid and not void. This is an illustration of another strength of our model where a single phase field represents the three materials. As far as we know, this kind of design has not been described in the literature. Notice that it is indeed very similar to an engineering technique in which one stiffens underground walls with cable and anchors instead of inner reinforcement. Also, as one would expect, every surface of the structured on which a pressure force is applied is arch-shaped.

Also, the design has no sharp corners, as it is usually the case in perimeter-controlled topology optimization. A heuristic explanation is that "rounding" a corner inside of a ball of radius $h$ requires using an amount of material proportional to $\pm h^{N}$ while it always diminishes the perimeter of an amount of the order of $h^{N-1}$. Therefore, there might exist a critical radius under which a sharp corner is not optimal. Unfortunately, a rigorous analyze of this kind of geometric effects would require a knowledge of the regularity of the optimal designs that is beyond the scope of this paper.

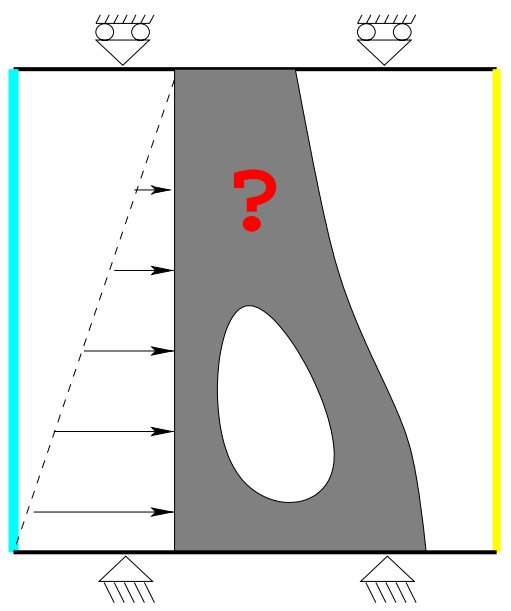

(a) Schematic problem

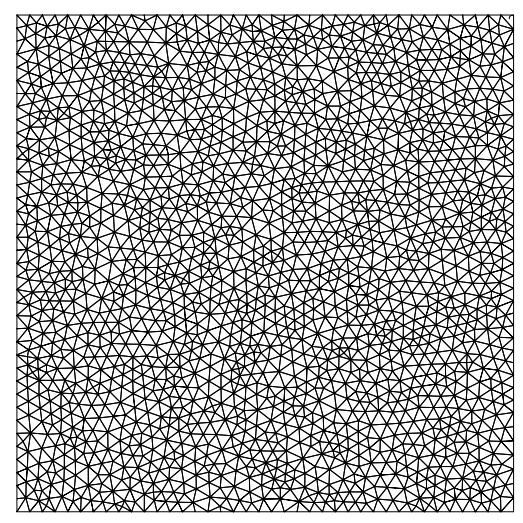

(b) Discretization of the domain

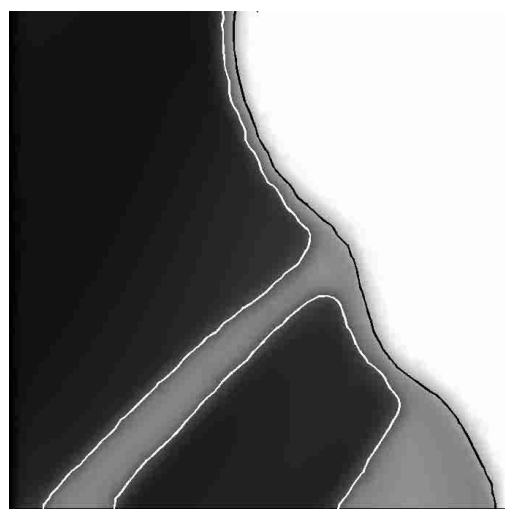

(c) Optimal design, $12 \%$ of material

Figure 3. Optimal design of a dam.

\footnotetext{
${ }^{5}$ Available at http://www-rocq.inria.fr/gamma/cdrom/www/emc2/eng.htm
} 
Remark 2.7. Prescribing the normal displacement of the upper part of the domain is quite unrealistic. However due to the hypothesis $(\mathrm{H})$ on the boundary conditions (see p. 22) we need to prescribe the phase or the normal displacement at each point of $\partial \Omega$. We chose to fix the latter and to leave as much freedom on the design as possible. Also, the restriction that $p$ should not vanish is not satisfied on the upper edge of the domain. That means that the liquid and void phases could be in contact with each other at the top of the computational design and that some part of the very thin ligament might actually not represent the structure itself but this interface (see the discussion in Sect. 5). However, even if these reasons make this simulation somehow unrealistic, we believe that it illustrates some interesting features of the method.

\subsubsection{Design of a cork}

The second example presented here is the optimal design of a cork (or a piston). The computational domain is the rectangle $(0,1) \times(-.8, .3)$ from which a smaller rectangle (shown in black in Fig. $4 \mathrm{a}),(0, .05) \times(-.05, .05)$ has been removed. On the sides of the smaller rectangle, one fixes both components of the displacement field while on the vertical edges of the domain, the normal displacement is held fixed. On the upper and lower edges of the domain, the displacement field is left free but the phase field is forced to take respectively the value -1 (for the void) and 1 (for the liquid).

As the normal displacement is fixed on both side of the domain, both can be identified as symmetry axis for a larger domain. If one uses the right edge, the simulation is related to that of a cork closing a pipe. Otherwise, it can be seen as a piston free to move between two walls. Here, the approximation parameter has been fixed $(\varepsilon \simeq 1.6)$, and the convergence is much slower than in the previous simulation (typically about 10000 iteration with a stopping criterion of $\left.3 \times 10^{-4}\right)$ despite the fewer number of degrees of freedom (1485 nodes and 2829 elements). The weight on the perimeter is set to $5 \times 10^{-4}$, and the step $r$ of the semi-implicit descent scheme is gradually increased from $10^{-3}$ to $6 \times 10^{-3}$. The volume fraction of the structure is set to $12 \%$. Figures 4b, 4c, and 4d use the same color scheme as before and differ from the choice of the magnitude of the pressure field, set equal to $10^{-1}$ in Figure $4 \mathrm{~b}, 1.4 \times 10^{-1}$ in Figure $4 \mathrm{c}$ and $1.5 \times 10^{-1}$ in Figure $4 \mathrm{~d}$. The numerical values of the compliance and the surface energy are $C \simeq 8.7 \times 10^{-2}$ and $\lambda \Lambda \simeq 2.2 \times 10^{-2}$ in Figure $4 \mathrm{~b}$, $C \simeq .17$ and $\lambda \Lambda \simeq 3.3 \times 10^{-2}$ in Figure $4 \mathrm{c}$, and $C \simeq .19$ and $\lambda \Lambda \simeq 4 \times 10^{-3}$ in Figure $4 \mathrm{~d}$

Remark that the problem is unchanged if one modifies the parameter $\lambda$ and $p$ in such a way that $p \lambda$ remains constant (so the the ratio between compliance and surface energy is unmodified). Hence, this set of figures also illustrates how the complexity of the optimal structures increases when the weight on the perimeter is lowered.

Again, the comments stated in the previous simulation, pertaining to the absence of cusps and the arch-shaped members, apply.

\section{Convergence of the Phase-Field approximation}

The proof of the approximation Theorem 1.5, relies on the basic properties of the $\Gamma$-convergence, recalled in Appendix A. First, we prove an approximation and a compactness theorem for the surface energy. Then, we show that the compliance is a continuous function of the design. This allows us to deduce the convergence of the phase-field model to the fictitious material one, which yields easily to the desired result.

\subsection{The approximation of the perimeter}

As a first step toward the proof of Theorem 1.5, we need to prove an approximation result for the surface term in the objective function, under the volume constraints. For that, we introduce a variant of the functional $\Lambda_{\varepsilon}$ introduced in Section 1.4.

Given a set of proportions $\theta=\left(\theta_{V}, \theta_{S}, \theta_{L}\right)=\left(\theta_{\alpha}\right)_{\alpha=-1,0,1}$, with $\theta_{\alpha} \in(0,1)$ for every $\alpha$ and $\theta_{-1}+\theta_{0}+\theta_{1}=1$, we define the functional $\bar{\Lambda}^{\theta}(\rho)=\bar{\Lambda}(\rho)$ if $|\{\rho=\alpha\}|=\theta_{\alpha}|\Omega|$ for each phase $\alpha$, and $\bar{\Lambda}^{\theta}(\rho)=+\infty$ otherwise. Also, for every $\rho \in L^{1}(\Omega)$ we define the functional $\Lambda_{\varepsilon}^{\theta}(\rho)=\Lambda_{\varepsilon}(\rho)$ if $\int_{\Omega} L(\rho(x)) \mathrm{d} x=\theta_{1}|\Omega|$ and $\int_{\Omega} V(\rho(x)) \mathrm{d} x=\theta_{-1}|\Omega|$, and $\Lambda_{\varepsilon}^{\theta}(\rho)=+\infty$. 


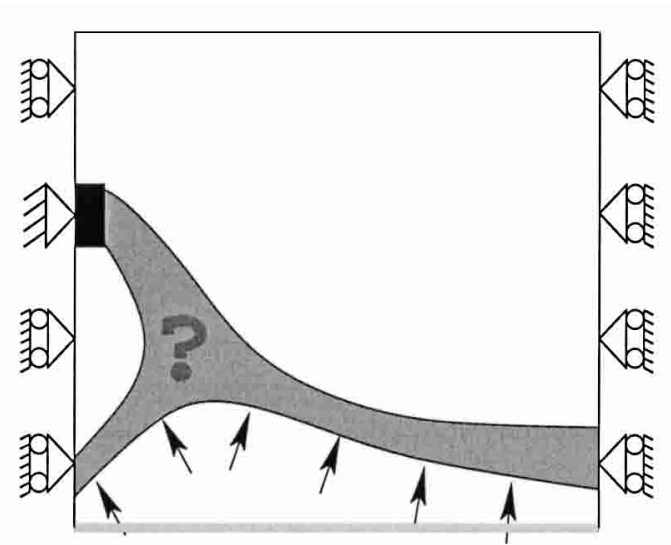

(a) Schematic problem

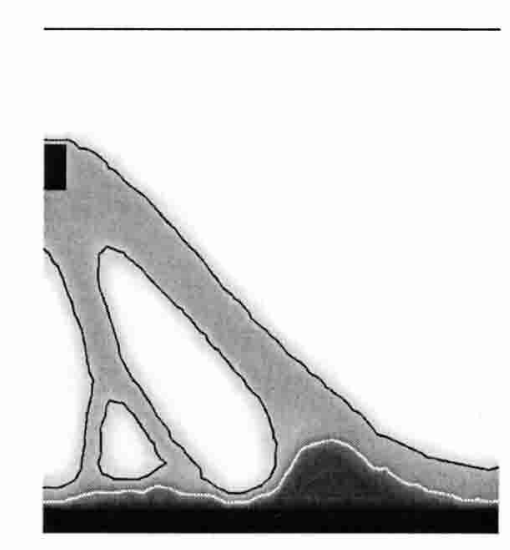

(c) $p \simeq 1.410^{-1}$

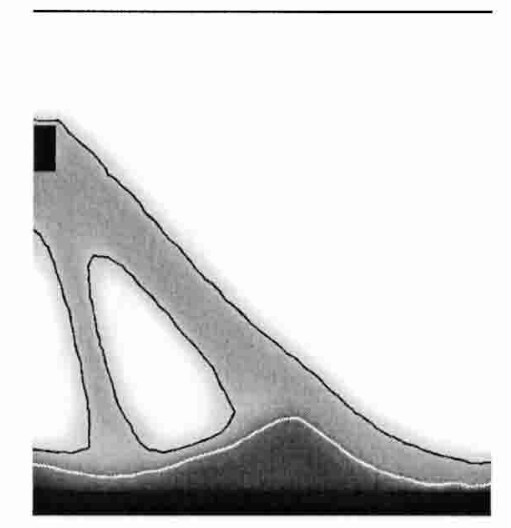

(b) $p \simeq 10^{-1}$

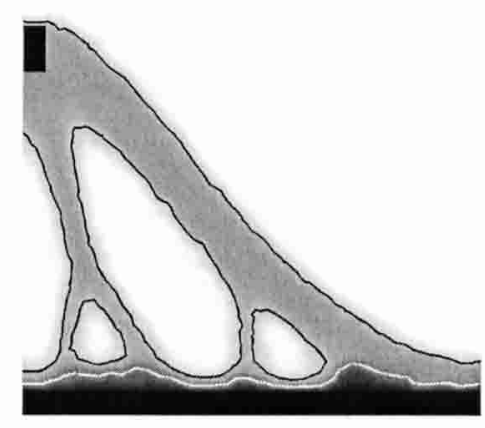

(d) $p \simeq 1.510^{-1}$

FiguRE 4. Optimal design of a piston for increasing values of the pressure field.

Then, we are able to state the following approximation theorem, which is a slightly modified version of a result due to Modica and Mortola [32,33] (see also [1]).

Theorem 3.1. As $\varepsilon$ goes to zero, $\Lambda_{\varepsilon} \Gamma$-converges to $\bar{\Lambda}$ in $L^{1}(\Omega)$, and $\Lambda_{\varepsilon}^{\theta} \Gamma$-converges to $\bar{\Lambda}^{\theta}$. Moreover, if the functions $\rho_{\varepsilon}$ are such that $\sup _{\varepsilon>0} \Lambda_{\varepsilon}\left(\rho_{\varepsilon}\right)<+\infty$, then there exists a sequence $\varepsilon_{j}$ going to 0 and $\rho \in X_{\rho}$ such that $\rho_{\varepsilon_{j}} \rightarrow \rho$ in $L^{1}(\Omega)$ as $j \rightarrow \infty$.

Proof. This result is very similar to the Theorem 2 in [33], which is also proved with a different method in [1]. The only differences between our Theorem 3.1 and the above mentioned results lie in the constraints on the proportion of each phase, and in the fact that we want to impose a Dirichlet boundary condition on the phase $\rho$. For the second point, it means that given $\rho \in \bar{X}_{\rho}$, and given a sequence $\left(\varepsilon_{j}\right)_{j \geq 1}$ with $\varepsilon_{j} \downarrow 0$ as $j \rightarrow \infty$, we must be able to build a family $\left(\rho_{j}\right)_{j \geq 1}$ of functions in $X_{\rho}$ such that $\rho_{j} \rightarrow \rho$ and

$$
\limsup _{j \rightarrow \infty} \Lambda_{\varepsilon_{j}}\left(\rho_{j}\right) \leq \bar{\Lambda}(\rho) .
$$


In order to do this we first observe that we may find a sequence $\left(\rho^{n}\right)_{n \geq 1}$ of functions in $B V\left(\Omega \cup \Gamma^{\rho} ;\{-1,0,1\}\right)$ such that each $\rho^{n}$ is identically $\alpha$ in a neighborhood of $\Gamma_{\alpha}^{\rho}, \alpha=-1,0,1$ (so that for every $n, \bar{\Lambda}\left(\rho^{n}\right)=\left|D \rho^{n}\right|(\Omega)$ ) and $\limsup _{n \rightarrow \infty}\left|D \rho^{n}\right|(\Omega)=\bar{\Lambda}(\rho)$. Each function $\rho^{n}$ may be defined by letting $\rho^{n}=\rho$ in $\Omega$ except $\rho^{n}=\alpha$ in $\left\{\operatorname{dist}\left(x, \Gamma_{\alpha}^{\rho}\right)<\delta / n\right\}$ for an appropriately chosen $\delta>0$, small, and this can be done because we have assumed that $\operatorname{dist}\left(\Gamma_{\alpha}^{\rho}, \Gamma_{\alpha^{\prime}}^{\rho}\right)>0$ if $\alpha \neq \alpha^{\prime}$. This is equivalent to showing that $\bar{\Lambda}$ is the lower semicontinuous envelope of $\Lambda$, see Appendix B for details.

Then, the construction of a sequence $\rho_{j}^{n}$ converging in $L^{1}(\Omega)$ to such a $\rho^{n}$ and satisfying $\lim \sup _{j \rightarrow \infty} \Lambda_{\varepsilon_{j}}\left(\rho_{j}^{n}\right)$ $\leq\left|D \rho^{n}\right|(\Omega)$, is the same as in [33], and we easily can adapt this construction in order to make sure that the functions $\rho_{j}^{n}$ satisfy the Dirichlet boundary conditions on $\Gamma^{\rho}\left(\rho_{j}^{n} \in X_{\rho}\right)$. Eventually, a standard diagonalization argument allows to build out of the sequences $\left(\rho_{j}^{n}\right)_{j \geq 1}, n \geq 1$, a sequence $\left(\rho_{j}\right)$ that converges to $\rho$ and satisfies (30).

Once the sequence $\left(\rho_{j}\right)$ has been built, we notice that $\lim _{j \rightarrow \infty} \int_{\Omega} L\left(\rho_{j}(x)\right) \mathrm{d} x=|\{\rho=1\}|=\theta_{1}|\Omega|$, and $\lim _{j \rightarrow \infty} \int_{\Omega} V\left(\rho_{j}(x)\right) \mathrm{d} x=|\{\rho=-1\}|=\theta_{-1}|\Omega|$. Therefore we may perturb slightly (at least for $j$ large enough) the functions $\rho_{j}$ in order to get the exact equalities $\int_{\Omega} L\left(\rho_{j}(x)\right) \mathrm{d} x=\theta_{1}|\Omega|, \int_{\Omega} V\left(\rho_{j}(x)\right) \mathrm{d} x=\theta_{-1}|\Omega|$ without perturbing much the energy $\Lambda_{\varepsilon_{j}}$ nor changing the limit $\rho$. This is done, for instance, by Baldo in [6] (see also [7]).

Remark 3.2. In this theorem, we have used the phase indicators $S, L, V$ introduced in Section 1.4 for the penalization of the volume fraction. It is however obvious that any other set of functions with a similar behaviour could be used instead

\subsection{Continuity of the compliance}

We now study the functional (18). First we notice that for any $\rho \in L^{1}(\Omega), 0 \leq C_{\delta}(\rho)<+\infty$, and the inf in (18) is in fact a min. Indeed, since $u \in X_{u}$ satisfies $u=0$ on some fixed non negligible part of the boundary $\partial \Omega$, there exists by Korn's inequality (since $\partial \Omega$ is Lipschitz-regular, see [19], Th. 6.3-4 or [39]) a constant $c_{K}$ such that

$$
\|u\|_{H^{1}\left(\Omega ; \mathbb{R}^{N}\right)}^{2} \leq c_{K} \int_{\Omega} A \mathrm{e}(u)(x): \mathrm{e}(u)(x) \mathrm{d} x
$$

for every $u \in X_{u}$. We deduce that the energy $E_{\delta}(\rho, u)$ is coercive in $u$, uniformly in $\rho$ : for every $u \in X_{u}$ and $\rho \in L^{1}(\Omega)$,

$$
\begin{aligned}
E_{\delta}(\rho, u) & \geq \frac{\delta}{2 c_{K}}\|u\|_{H^{1}\left(\Omega ; \mathbb{R}^{N}\right)}^{2}-\|f\|_{L^{2}\left(\Omega ; \mathbb{R}^{N}\right)}\|u\|_{L^{2}\left(\Omega ; \mathbb{R}^{N}\right)}-\|p\|_{H^{1}\left(\Omega ; \mathbb{R}^{N}\right)}\|u\|_{H^{1}\left(\Omega ; \mathbb{R}^{N}\right)}-\|p\|_{L^{2}\left(\Gamma^{\rho}\right)}\|u\|_{L^{2}\left(\Gamma^{\rho} ; \mathbb{R}^{N}\right)} . \\
& \geq c_{1}\|u\|_{H^{1}\left(\Omega ; \mathbb{R}^{N}\right)}^{2}-c_{2}
\end{aligned}
$$

with two constants $c_{1}$ and $c_{2}$ depending only on $\delta$. In particular, we easily deduce that the infimum problem in (18) always has a solution $u_{\rho}$, characterized by

$$
\begin{aligned}
\int_{\Omega}(\delta+(1-\delta) S(\rho(x))) A \mathrm{e}\left(u_{\rho}\right)(x): \mathrm{e}(v)(x) \mathrm{d} x= & \int_{\Omega} S(\rho(x)) f(x) \cdot v(x) \mathrm{d} x+\int_{\Omega} L(\rho(x)) \operatorname{div}(p v)(x) \mathrm{d} x \\
& -\int_{\Gamma_{1}^{\rho}} p(x) v(x) \cdot \nu_{\Omega}(x) \mathrm{d} \mathcal{H}^{N-1}(x)
\end{aligned}
$$

for every $v \in X_{u}$, so that $C_{\delta}(\rho)<+\infty$ for every $\rho \in L^{1}(\Omega)$. Letting $v=u_{\rho}$ in $(33)$, we discover that

$$
C_{\delta}(\rho)=\int_{\Omega} S(\rho(x)) f(x) \cdot u(x) \mathrm{d} x+\int_{\Omega} L(\rho(x)) \operatorname{div}(p u)(x) \mathrm{d} x-\int_{\Gamma_{1}^{\rho}} p(x) u(x) \cdot \nu_{\Omega}(x) \mathrm{d} \mathcal{H}^{N-1}(x)
$$


and if $\rho \in \bar{X}_{\rho}$,

$$
C_{\delta}(\rho)=\int_{\Omega} S(\rho(x)) f(x) \cdot u_{\rho}(x) \mathrm{d} x-\int_{\Omega \cup \Gamma^{\rho}} p(x) u_{\rho}(x) \cdot D L(\rho),
$$

which actually corresponds to the total work of the forces at equilibrium.

We then can show the following lemma:

Lemma 3.3. The functional $\rho \mapsto C_{\delta}(\rho)$ is (strongly) continuous in $L^{1}(\Omega)$.

Proof. Consider a sequence $\left(\rho_{n}\right)_{n \geq 1}$ that converges to some $\rho$ in $L^{1}(\Omega)$. Without loss of generality we may assume that the sequence $\left(C_{\delta}\left(\rho_{n}\right)\right)_{n \geq 1}$ converges to some limit $\ell \in[0,+\infty]$. For every $n$ let $u_{n} \in X_{u}$ the minimizer of $E_{\delta}\left(\rho_{n}, \cdot\right)$ over $X_{u}$.

By (32), we see that the sequence $\left(u_{n}\right)_{n \geq 1}$ is bounded in $H^{1}\left(\Omega ; \mathbb{R}^{N}\right)\left(\left\|u_{n}\right\|_{H^{1}\left(\Omega ; \mathbb{R}^{N}\right)}^{2} \leq c_{2} / c_{1}\right)$. We may extract a subsequence $\left(u_{n_{k}}\right)$ converging weakly in $H^{1}$ to some $u \in X_{u}$. Since $S\left(\rho_{n}\right) \rightarrow S(\rho)$ strongly (in any $L^{p}(\Omega)$, $p<+\infty)$, and $L\left(\rho_{n}\right) \rightarrow L(\rho)$, taking $\rho=\rho_{n_{k}}$ and $u_{\rho}=u_{n_{k}}$ in (33) and passing to the limit we see that $u$ is the minimizer of $E_{\delta}(\rho, \cdot)$ over $X_{u}$.

Now, we also have that (by (34))

$$
C_{\delta}\left(\rho_{n_{k}}\right)=\int_{\Omega} S\left(\rho_{n_{k}}(x)\right) f(x) \cdot u_{n_{k}}(x) \mathrm{d} x+\int_{\Omega} L\left(\rho_{n_{k}}(x)\right) \operatorname{div}\left(p u_{n_{k}}\right)(x) \mathrm{d} x-\int_{\Gamma_{1}^{\rho}} p(x) u_{n_{k}}(x) \cdot \nu_{\Omega}(x) \mathrm{d} \mathcal{H}^{N-1}(x) .
$$

Since $u_{n_{k}}$ goes to $u$ strongly in $L^{2}(\partial \Omega)$ we can pass to the limit in this expression and get that

$$
\begin{aligned}
\ell & =\int_{\Omega} S(\rho(x)) f(x) \cdot u(x) \mathrm{d} x+\int_{\Omega} L(\rho(x)) \operatorname{div}(p u)(x) \mathrm{d} x-\int_{\Gamma_{1}^{\rho}} p(x) u(x) \cdot \nu_{\Omega}(x) \mathrm{d} \mathcal{H}^{N-1}(x) . \\
& =C_{\delta}(\rho)
\end{aligned}
$$

in particular, $\ell<+\infty$, and Lemma 3.3 is proved.

From Theorem 3.1 and Lemma 3.3 we deduce the following corollary:

Corollary 3.4. As $\varepsilon$ goes to zero, the functional $C_{\delta}+\lambda \Lambda_{\varepsilon} \Gamma$-converges in $L^{1}(\Omega)$ to $C_{\delta}+\lambda \bar{\Lambda}$, and (given a set of proportions $\left.\theta=\left(\theta_{-1}, \theta_{0}, \theta_{1}\right) \in(0,1)^{3}, \theta_{-1}+\theta_{0}+\theta_{1}=1\right) C_{\delta}+\lambda \Lambda_{\varepsilon}^{\theta} \Gamma$-converges to $C_{\delta}+\lambda \bar{\Lambda}^{\theta}$.

We deduce Theorem 1.5 from this corollary: if $\rho_{\varepsilon}$ minimizes $C_{\delta}+\lambda \Lambda_{\varepsilon}^{\theta}$ in $L^{1}(\Omega)$ (in fact, in $X_{\rho}$ ), then by Theorem 3.1 there exists $\rho \in \bar{X}_{\rho}$ and a sequence $\varepsilon_{j}$ going to 0 as $j \rightarrow \infty$ such that $\rho_{\varepsilon_{j}} \rightarrow \rho$, and by Corollary 3.4 $\rho$ is a minimizer of $C_{\delta}+\lambda \bar{\Lambda}^{\theta}$, in other words a solution of (19).

\section{Analysis and limit of the fictitious material Problem}

\subsection{The dual problem}

In order to study the limit $\delta \rightarrow 0$, we have to introduce (exactly like Allaire et al. in [2] in a similar situation) the dual problem of (18). To define the dual problem we follow ([21], Chap. III).

The compliance $C_{\delta}(\rho)$ that we minimize is given by (18). We introduce as in [21] the perturbed energy $(\delta>0$ and $\rho \in L^{1}(\Omega)$ being fixed)

$$
\begin{aligned}
\Phi_{\delta, \rho}(u, q)= & \frac{1}{2} \int_{\Omega}(\delta+(1-\delta) S(\rho(x))) A(\mathrm{e}(u)(x)+q(x)):(\mathrm{e}(u)(x)+q(x)) \mathrm{d} x \\
& -\int_{\Omega} S(\rho(x)) f(x) \cdot u(x)-\int_{\Omega} L(\rho(x)) \operatorname{div}(p u)(x) \mathrm{d} x+\int_{\Gamma_{1}^{\rho}} p(x) u(x) \cdot \nu_{\Omega}(x) \mathrm{d} \mathcal{H}^{N-1}(x),
\end{aligned}
$$


defined for $u \in X_{u}$ and $q \in L^{2}\left(\Omega ; \mathcal{S}^{N}\right)$ where $\mathcal{S}^{N} \simeq \mathbb{R}^{N(N+1) / 2}$ is the set of symmetric $N \times N$ matrices. The dual problem of (18) is by definition the problem

$$
\sup _{\sigma \in L^{2}\left(\Omega ; \mathcal{S}^{N}\right)}\left\{-\Phi_{\delta, \rho}^{*}(0, \sigma)\right\}
$$

where $\Phi_{\delta, \rho}^{*}$ is the Legendre-Fenchel conjugate of $\Phi_{\delta, \rho}$. This conjugate is given by

$$
\begin{aligned}
c \Phi_{\delta, \rho}^{*}(0, \sigma)= & \sup _{u \in X_{u}, q \in L^{2}\left(\Omega ; \mathcal{S}^{N}\right)} \int_{\Omega} q: \sigma \mathrm{d} x-\Phi_{\delta, \rho}(u, q) \\
= & \sup _{u \in X_{u}}\left(\sup _{q \in L^{2}\left(\Omega ; \mathcal{S}^{N}\right)} \int_{\Omega} q: \sigma d x-\frac{1}{2} \int_{\Omega}(\delta+(1-\delta) S(\rho)) A(\mathrm{e}(u)+q):(\mathrm{e}(u)+q) \mathrm{d} x\right) \\
& +\int_{\Omega} S(\rho) f \cdot u+L(\rho) \operatorname{div}(p u) \mathrm{d} x-\int_{\Gamma_{1}^{\rho}} p u \cdot \nu_{\Omega} \mathrm{d} \mathcal{H}^{N-1}, \\
= & \frac{1}{2} \int_{\Omega} \frac{1}{\delta+(1-\delta) S(\rho)} A^{-1} \sigma: \sigma \mathrm{d} x \\
& +\sup _{u \in X_{u}} \int_{\Omega}-\mathrm{e}(u): \sigma+S(\rho) f \cdot u+L(\rho) \operatorname{div}(p u) \mathrm{d} x-\int_{\Gamma_{1}^{\rho}} p u \cdot \nu_{\Omega} \mathrm{d} \mathcal{H}^{N-1} .
\end{aligned}
$$

Introducing thus the functional $F_{\delta}(\rho, \sigma)=\Phi_{\delta, \rho}^{*}(0, \sigma)$ :

$$
F_{\delta}(\rho, \sigma)=\frac{1}{2} \int_{\Omega} \frac{1}{\delta+(1-\delta) S(\rho(x))} A^{-1} \sigma(x): \sigma(x) \mathrm{d} x,
$$

if $\forall u \in X_{u}$,

$$
\int_{\Omega}-\mathrm{e}(u): \sigma+S(\rho) f \cdot u+L(\rho) \operatorname{div}(p u) \mathrm{d} x-\int_{\Gamma_{1}^{\rho}} p u \cdot \nu_{\Omega} \mathrm{d} \mathcal{H}^{N-1}=0,
$$

and $F_{\delta}(\rho, \sigma)=+\infty$ otherwise, the dual problem of (18) may be restated as

$$
C_{\delta}(\rho)=2 \inf _{\sigma \in L^{2}\left(\Omega ; \mathcal{S}^{N}\right)} F_{\delta}(\rho, \sigma) .
$$

The equivalence of the two problems (18) and (37) (and the equality in (37)) comes from the fact that problem (18) is normal ([21], Def. III-2.1). This follows from ([21], Th. III-4.1). From the latter theorem we also deduce that problem (37) admits a minimizer $\sigma_{\rho}$.

\subsection{Convergence of the fictitious material model}

Consider now the functional, for $\delta>0$, and $\rho \in L^{1}(\Omega), \sigma \in L^{2}\left(\Omega ; \mathcal{S}^{N}\right), \mathcal{F}_{\delta}(\rho, \sigma)=2 F_{\delta}(\rho, \sigma)+\lambda \bar{\Lambda}(\rho)$, and (given a set of proportions $\left.\theta=\left(\theta_{-1}, \theta_{0}, \theta_{1}\right) \in(0,1)^{3}, \theta_{-1}+\theta_{0}+\theta_{1}=1\right)$ the functional $\mathcal{F}_{\delta}^{\theta}(\rho, \sigma)$ $=2 F_{\delta}(\rho, \sigma)+\lambda \bar{\Lambda}^{\theta}(\rho)$.

Define, for $\rho \in \bar{X}_{\rho}\left(\rho \in B V\left(\Omega \cup \Gamma^{\rho} ;\{-1,0,1\}\right)\right)$, and $\sigma \in L^{2}\left(\Omega ; \mathcal{S}^{N}\right)$, the functional

$$
\begin{aligned}
F_{0}(\rho, \sigma)= & \frac{1}{2} \int_{S(\rho)} A^{-1} \sigma(x): \sigma(x) \mathrm{d} x, \\
& \text { if } \sigma=0 \text { a.e. in } V(\rho) \cup L(\rho)=S(\rho)^{c} \text { and } \forall u \in X_{u},(36) \text { holds, }
\end{aligned}
$$

and $F_{0}(\rho, \sigma)=+\infty$ otherwise. 
Notice that for $\rho \in \bar{X}_{\rho}$, condition (36) may also be written

$$
\forall u \in X_{u}, \int_{\Omega}-\mathrm{e}(u): \sigma+S(\rho) f \cdot u \mathrm{~d} x-\int_{\Omega \cup \Gamma^{\rho}} p u \cdot D L(\rho)=0,
$$

and is a weak way of expressing the fact that in $\Omega$,

$$
\operatorname{div} \sigma+S(\rho) f-p D L(\rho)=0
$$

while $\sigma \cdot \nu_{\Omega}+p \nu_{L}=0$ on $\bar{\partial}_{\star} L(\rho) \cap \partial \Omega \backslash \Gamma^{u}$. We then set $\mathcal{F}_{0}(\rho, \sigma)=2 F_{0}(\rho, \sigma)+\lambda \bar{\Lambda}(\rho)$ and $\mathcal{F}_{0}^{\theta}(\rho, \sigma)$ $=2 F_{0}(\rho, \sigma)+\lambda \bar{\Lambda}^{\theta}(\rho)$. We have the following theorem:

Theorem 4.1. As $\delta \rightarrow 0$, $\mathcal{F}_{\delta} \Gamma$-converges to $\mathcal{F}_{0}$ in $L^{1}(\Omega) \times\left(L^{2}\left(\Omega ; \mathcal{S}^{N}\right)\right.$-weak $)$, and $\mathcal{F}_{\delta}^{\theta} \Gamma$-converges to $\mathcal{F}_{0}^{\theta}$. Moreover if for $\delta>0$ small, $\mathcal{F}_{\delta}\left(\rho_{\delta}, \sigma_{\delta}\right) \leq c<+\infty$, then there exist $\rho, \sigma$ and a sequence $\left(\delta_{j}\right)_{j \geq 1}$ (with $\delta_{j} \downarrow 0$ as $j \rightarrow \infty)$ such that $\rho_{\delta_{j}} \rightarrow \rho$ in $L^{1}(\Omega)$ and $\sigma_{\delta_{j}} \rightarrow \sigma$ weakly in $L^{2}\left(\Omega ; \mathcal{S}^{N}\right)$ as $j \rightarrow \infty$.

Proof. Consider first (for $\delta>0$ small) functions $\rho_{\delta}, \sigma_{\delta}$ such that $\mathcal{F}_{\delta}\left(\rho_{\delta}, \sigma_{\delta}\right) \leq c<+\infty$ : we will show that there exist $\rho_{0}, \sigma_{0}$ and $\left(\delta_{j}\right)_{j \geq 1}$ with $\rho_{\delta_{j}} \rightarrow \rho_{0}, \sigma_{\delta_{j}} \rightarrow \sigma_{0}$, and $\mathcal{F}_{0}\left(\rho_{0}, \sigma_{0}\right) \leq \liminf _{j \rightarrow \infty} \mathcal{F}_{\delta_{j}}\left(\rho_{\delta_{j}}, \sigma_{\delta_{j}}\right)$. Notice first that since $\bar{\Lambda}\left(\rho_{\delta}\right)=\left|D \rho_{\delta}\right|\left(\Omega \cup \Gamma^{\rho}\right)$ is uniformly bounded, there exist a sequence $\left(\delta_{j}\right)_{j \geq 1}$ and $\rho_{0}$ such that $\rho_{\delta_{j}} \rightarrow \rho_{0}$ in $L^{1}(\Omega)$, and $\bar{\Lambda}\left(\rho_{0}\right)=\left|D \rho_{0}\right|\left(\Omega \cup \Gamma^{\rho}\right) \leq \liminf _{j \rightarrow \infty} \bar{\Lambda}\left(\rho_{\delta_{j}}\right)$. To simplify the notations we let $\rho_{j}=\rho_{\delta_{j}}$ and $\sigma_{j}=\sigma_{\delta_{j}}$. We may assume without loss of generality that $\rho_{j}$ converges to $\rho$ a.e. in $\Omega$.

Now, we have that

$$
F_{\delta}\left(\rho_{\delta}, \sigma_{\delta}\right)=\frac{1}{2 \delta} \int_{S\left(\rho_{\delta}\right)^{c}} A^{-1} \sigma_{\delta}: \sigma_{\delta} \mathrm{d} x+\frac{1}{2} \int_{S\left(\rho_{\delta}\right)} A^{-1} \sigma_{\delta}: \sigma_{\delta} \mathrm{d} x \leq c<+\infty
$$

$\left(S\left(\rho_{\delta}\right)^{c}=V\left(\rho_{\delta}\right) \cup L\left(\rho_{\delta}\right)\right)$ so that $\sigma_{\delta}$ is bounded in $L^{2}\left(\Omega ; \mathcal{S}^{N}\right)$ and we may therefore assume that there exists $\sigma_{0}$ such that $\sigma_{j} \rightarrow \sigma_{0}$ weakly in $L^{2}$. We introduce the functions $\sigma_{j}^{\prime}=S\left(\rho_{j}\right) \sigma_{j}$ and $\sigma_{j}^{\prime \prime}=\sigma_{j}-\sigma_{j}^{\prime}=\left(1-S\left(\rho_{j}\right)\right) \sigma_{j}$. The estimate (39) yields

$$
\int_{\Omega} A^{-1} \sigma_{j}^{\prime \prime}: \sigma_{j}^{\prime \prime} \mathrm{d} x \leq 2 c \delta_{j},
$$

therefore $\sigma_{j}^{\prime \prime} \rightarrow 0$ strongly in $L^{2}$. We deduce that $\sigma_{j}^{\prime} \rightarrow \sigma_{0}$, and in particular that

$$
\frac{1}{2} \int_{\Omega} A^{-1} \sigma_{0}: \sigma_{0} \mathrm{~d} x \leq \liminf _{j \rightarrow \infty} \frac{1}{2} \int_{\Omega} A^{-1} \sigma_{j}^{\prime}: \sigma_{j}^{\prime} \mathrm{d} x \leq \liminf _{j \rightarrow \infty} F_{\delta_{j}}\left(\rho_{j}, \sigma_{j}\right) .
$$

To conclude that $\mathcal{F}_{0}\left(\rho_{0}, \sigma_{0}\right) \leq \liminf _{j \rightarrow \infty} \mathcal{F}_{\delta_{j}}\left(\rho_{j}, \sigma_{j}\right)$ it remains to show that $\sigma_{0}=0$ a.e. in $S\left(\rho_{0}\right)^{c}$ and that for every $u \in X_{u}$, equation (36) holds for $\sigma=\sigma_{0}, \rho=\rho_{0}$.

The fact that $\sigma_{0}=0$ a.e. in $S\left(\rho_{0}\right)^{c}$ is clear because for every $\varphi \in L^{2}\left(\Omega, \mathcal{S}^{N}\right)$ with $\varphi=0$ a.e. in $S\left(\rho_{0}\right)$, $\varphi S\left(\rho_{j}\right) \rightarrow 0$ strongly in $L^{2}\left(\Omega, \mathcal{S}^{N}\right)$, thus $\int_{\Omega} \sigma_{j}^{\prime}: \varphi \mathrm{d} x=\int_{\Omega} \sigma_{j}:\left(\varphi S\left(\rho_{j}\right)\right) \mathrm{d} x \rightarrow 0$, but since $\sigma_{j}^{\prime} \rightarrow \sigma_{0}$ it means that $\int_{\Omega} \sigma_{0}: \varphi \mathrm{d} x=0$.

On the other hand, the fact that given $u \in X_{u}(36)$ holds is also elementary since $\left(F_{\delta_{j}}\left(\rho_{j}, \sigma_{j}\right)\right.$ being finite for every $j$ ) it holds for $\sigma=\sigma_{j}, \rho=\rho_{j}$, and passes to the limit as $j \rightarrow \infty$.

In order to achieve the proof of Theorem 4.1 we need, given $\rho_{0}, \sigma_{0}$, to find $\rho_{\delta}$ and $\sigma_{\delta}$ such that $\rho_{\delta} \rightarrow \rho_{0}$ in $L^{1}$, $\sigma_{\delta} \rightarrow \sigma_{0}$ in $L^{2}$, and $\lim \sup _{\delta \downarrow 0} \mathcal{F}_{\delta}\left(\rho_{\delta}, \sigma_{\delta}\right) \leq \mathcal{F}_{0}\left(\rho_{0}, \sigma_{0}\right)$. But since, if $\mathcal{F}_{0}\left(\rho_{0}, \sigma_{0}\right)<+\infty, \mathcal{F}_{\delta}\left(\rho_{0}, \sigma_{0}\right)=\mathcal{F}_{0}\left(\rho_{0}, \sigma_{0}\right)$ for every $\delta>0$, we can take $\rho_{\delta}=\rho_{0}, \sigma_{\delta}=\sigma_{0}$.

Now we introduce the limit compliance (defined only for $\rho \in \bar{X}_{\rho}$ )

$$
C_{0}(\rho)=2 \inf _{\sigma \in L^{2}\left(\Omega ; \mathcal{S}^{N}\right)} F_{0}(\rho, \sigma) .
$$


From Theorem 4.1 follows the

Corollary 4.2. As $\delta$ goes to zero, the functional $C_{\delta}+\lambda \bar{\Lambda} \Gamma$-converges in $L^{1}(\Omega)$ to $C_{0}+\lambda \bar{\Lambda}$, and $C_{\delta}+\lambda \bar{\Lambda}^{\theta}$ $\Gamma$-converges to $C_{0}+\lambda \bar{\Lambda}^{\theta}$.

Proof. Consider $\rho_{\delta}$ such that $C_{\delta}\left(\rho_{\delta}\right)+\lambda \bar{\Lambda}\left(\rho_{\delta}\right) \leq c<+\infty$ for small $\delta>0$. Clearly $\left(\rho_{\delta}\right)_{\delta>0}$ is compact in $L^{1}(\Omega)$ and there exist $\left(\delta_{j}\right)_{j \geq 1}$ going to zero and $\rho_{0}$ such that $\rho_{\delta_{j}} \rightarrow \rho_{0}$ in $L^{1}(\Omega)$. We let $\rho_{j}=\rho_{\delta_{j}}$ and define $\sigma_{j}$ as the minimizer of (37) for $\rho=\rho_{j}$. Then from Theorem 4.1 we get that (up to a subsequence) $\sigma_{j}$ goes weakly to some $\sigma_{0}$ in $L^{2}$ and

$$
C_{0}\left(\rho_{0}\right)+\lambda \bar{\Lambda}\left(\rho_{0}\right) \leq 2 F_{0}\left(\rho_{0}, \sigma_{0}\right)+\lambda \bar{\Lambda}\left(\rho_{0}\right) \leq \liminf _{j \rightarrow \infty} 2 F_{\delta_{j}}\left(\rho_{j}, \sigma_{j}\right)+\lambda \bar{\Lambda}\left(\rho_{j}\right)=\liminf _{j \rightarrow \infty} C_{\delta_{j}}\left(\rho_{j}\right)+\lambda \bar{\Lambda}\left(\rho_{j}\right)
$$

On the other hand, given $\rho_{0}$ such that $C_{0}\left(\rho_{0}\right)+\lambda \bar{\Lambda}\left(\rho_{0}\right)<+\infty$, if for an arbitrary $\eta>0$, $\sigma^{\eta}$ is such that $2 F_{0}\left(\rho_{0}, \sigma^{\eta}\right) \leq C_{0}\left(\rho_{0}\right)+\eta$, then $C_{\delta}\left(\rho_{0}\right) \leq 2 F_{\delta}\left(\rho_{0}, \sigma^{\eta}\right)=2 F_{0}\left(\rho_{0}, \sigma^{\eta}\right) \leq C_{0}\left(\rho_{0}\right)+\eta$. Therefore $C_{\delta}\left(\rho_{0}\right) \leq C_{0}\left(\rho_{0}\right)$ for every $\delta>0$ and we find a family $\left(\rho_{\delta}\right)_{\delta>0}$ such that $\lim \sup _{\delta \downarrow 0} C_{\delta}\left(\rho_{\delta}\right)+\lambda \bar{\Lambda}\left(\rho_{\delta}\right) \leq C_{0}\left(\rho_{0}\right)+\lambda \bar{\Lambda}\left(\rho_{0}\right)($ or, as well, $\left.\lim \sup _{\delta \downarrow 0} C_{\delta}\left(\rho_{\delta}\right)+\lambda \bar{\Lambda}^{\theta}\left(\rho_{\delta}\right) \leq C_{0}\left(\rho_{0}\right)+\lambda \bar{\Lambda}^{\theta}\left(\rho_{0}\right)\right)$ just by letting $\rho_{\delta}=\rho_{0}$ for every $\delta$.

\subsection{Analysis of the limit problem}

To sum up, we have shown that the problem we are actually (numerically) computing is an approximation of the problem

$$
\min _{\rho \in \bar{X}_{\rho}} C_{0}(\rho)+\lambda \bar{\Lambda}(\rho)
$$

where $C_{0}$ is defined by (40). We would like to show that this is the same as problem (9), or, equivalently, that $C_{0}(\rho)$ defines the same functional as $C(S, L, V)$ in $(7)$ for $\{S, L, V\}=\{S(\rho), L(\rho), V(\rho)\}$. Then, Theorem 1.3 (and as a corollary Th. 1.2) would easily follow from Corollary 4.2. Indeed, the assumption that there exists $\{S, L, V\}$ with $C(S, L, V)<+\infty$ would mean that there exists some $\rho \in \bar{X}_{\rho}$ such that $C_{0}(\rho)<+\infty$. This would imply that the minimizers $\rho_{\delta}$ of $C_{\delta}+\lambda \bar{\Lambda}^{\theta}$ satisfy $\sup _{\delta>0} C_{\delta}\left(\rho_{\delta}\right)+\lambda \bar{\Lambda}^{\theta}\left(\rho_{\delta}\right)<+\infty$, and thus would yield the existence of subsequences of $\left(\rho_{\delta}\right)_{\delta>0}$ that converge in $L^{1}(\Omega)$ to some minimizer of $(41)$, showing Theorem 1.3 .

To address this problem we simply consider the functional $\Phi_{0, \rho}(u, q)$ defined in (35), this time for $\rho \in \bar{X}_{\rho}$ and $\delta=0$. A standard calculation shows that $\Phi_{0, \rho}^{*}(0, \sigma)=F_{0}(\rho, \sigma)$. Therefore problem (40) is the dual problem of

$$
-2 \inf _{u \in X_{u}} E_{0}(\rho, u)
$$

where $E_{0}(\rho, u)=\Phi_{0, \rho}(u, 0)$. But $E_{0}(\rho, u)$ is identical to $E(\{S(\rho), L(\rho), V(\rho)\}, u)$ defined in (8), thus problem (42) is equivalent to (7) and its value is exactly $C(S(\rho), L(\rho), V(\rho))$. Now, the functional $\Phi_{0, \rho}$ satisfies $\Phi_{0, \rho}(0,0)=0$ and $q \mapsto \Phi_{0, \rho}(0, q)$ is continuous in $L^{2}\left(\Omega ; \mathcal{S}^{N}\right)$, therefore we may apply [21] (Th. III-4.1) whenever the infimum in (42) is finite, to conclude that in this case, it is equal to $C_{0}(\rho)$. In addition ([21], Prop. III-1.2) shows that the value of $(42)$ (positive since $\left.E_{0}(\rho, 0)=0\right)$, is less than $(40)$, therefore it is finite if and only if $C_{0}(\rho)<+\infty$. It follows that the value of $(42)$ is always equal to $C_{0}(\rho)$, whether finite or infinite. In other words, we always have $C_{0}(\rho)=C(S(\rho), L(\rho), V(\rho))$. Hence (41) is the same as $(9)$ and Theorem 1.3 is true.

Remark 4.3. From [21] (Th. III-4.1) we also deduce that when $C_{0}(\rho)<+\infty$, (40) admits a minimizer $\sigma_{\rho}$. On the other hand, it is unlikely that (42) admits a minimizer in $X_{u}$ for arbitrary $\rho \in \bar{X}_{\rho}$, even of finite compliance $C_{0}(\rho)$. 


\section{INTERFACES OF FINITE COMPLIANCE CONFIGURATIONS}

Throughout the paper, we have made the "common sense" assumption that there are no Liquid-Void interfaces in finite compliance configurations. Formally, this is because along such an interface, the void opposes no resistance to the liquid and should therefore be sent to infinity, yielding an infinite compliance.

However, a rigorous study of this point is much more delicate and is discussed in the present section. In the general case, if $\overline{\partial_{*} S}$ is essentially closed (i.e., closed up to a set of zero $(N-1)$-dimensional measure), the non-existence result is easy to obtain. In dimension two though, the situation is slightly simpler. Lemma 5.1 in Appendix $\mathrm{C}$ permits to show that given any $\rho$ such that $C_{0}(\rho)<+\infty$, the Liquid-Void interface is essentially empty, without the above-mentioned closedness hypothesis.

These issues are discussed in the present section.

The energy $E_{0}(\rho, u)$ may be written $\left(\rho \in \bar{X}_{\rho}, u \in X_{u}\right)$

$$
E_{0}(\rho, u)=\frac{1}{2} \int_{S(\rho)} A \mathrm{e}(u): \mathrm{e}(u) \mathrm{d} x-\int_{S(\rho)} f \cdot u \mathrm{~d} x-\int_{\bar{\partial}_{\star} L(\rho)} p u \cdot \nu_{L} \mathrm{~d} \mathcal{H}^{N-1}(x)
$$

with $\nu_{L}$ the exterior normal to $L(\rho)$. The last term actually corresponds to a pressure $p(x)$ exerted from $L(\rho)$ onto its complement. A problem is that we would like theoretically this pressure to be exerted only on the structure $S(\rho)$ and not also on the void $V(\rho)$, in other words, the last integral should be taken only on $\bar{\partial}_{\star} L(\rho) \cap \bar{\partial}_{\star} S(\rho)$ This is not a problem if we can show that actually, $\bar{\partial}_{\star} L(\rho) \subseteq \bar{\partial}_{\star} S(\rho)$ (essentially, i.e. up to a $\mathcal{H}^{N-1}$-negligible set). We can give a complete answer to this point only in dimension $N=2$.

Assume that $\rho$ is such that $C_{0}(\rho)$ is finite (for instance, $\rho$ is a minimizer of $(41)$ ). The essential point is that, as pointed out in Remark 4.3, equation (40) admits a minimizer. This minimizer $\sigma_{\rho}$ satisfies (38), or

$$
\forall u \in X_{u}, \int_{S(\rho)}-\mathrm{e}(u): \sigma_{\rho}+f \cdot u \mathrm{~d} x-\int_{\bar{\partial}_{\star} L(\rho)} p u \cdot \nu_{L} \mathrm{~d} \mathcal{H}^{N-1}(x)=0 .
$$

We recall the assumption that $p$ does not vanish (either $p>0$ on $\bar{\Omega}$ or $p<0$ on $\bar{\Omega}$ ). In the case where $S(\rho)$ is a regular (say, at least Lipschitz) set, (43) means (if we integrate by part) that necessarily,

$$
\begin{cases}\operatorname{div} \sigma_{\rho}+f=0 & \text { in } S(\rho), \\ \bar{\partial}_{\star} L(\rho) \backslash \Gamma^{u} \subseteq \partial S(\rho) & \text { (essentially), } \\ \sigma_{\rho} \cdot \nu_{S}=p \nu_{S} & \mathcal{H}^{N-1} \text {-a.e. in } \bar{\partial}_{\star} L(\rho) \backslash \Gamma^{u}, \text { and } \\ \sigma_{\rho} \cdot \nu_{S}=0 & \mathcal{H}^{N-1} \text {-a.e. in }\left(\partial S(\rho) \backslash \bar{\partial}_{\star} L(\rho)\right) \backslash \Gamma^{u} .\end{cases}
$$

These are the classical expected conditions for the stress tensor $\sigma_{\rho}$.

If, on the other hand, we could show that the boundary $\tilde{\partial}_{\star} S(\rho)$ (where here $\tilde{\partial}_{\star} E$ denotes the measuretheoretical boundary of the finite-perimeter set $E$ seen as a subset of $\mathbb{R}^{N}$ rather than $\Omega$ ) is essentially closed (i.e., $\left.\left.\mathcal{H}^{N-1} \overline{\left(\tilde{\partial}_{\star} S(\rho)\right.} \backslash \tilde{\partial}_{\star} S(\rho)\right)=0\right)$, then using a blow-up argument around a point of $\bar{\partial}_{\star} L(\rho) \cap \partial_{\star} V(\rho) \backslash\left(\tilde{\partial}_{\star} S(\rho) \cup \Gamma^{u}\right)$ we would find again that the liquid-void interface is essentially empty: $\mathcal{H}^{N-1}\left(\bar{\partial}_{\star} L(\rho) \cap \partial_{\star} V(\rho)\right)=0$. We would deduce this time that $\bar{\partial}_{\star} L(\rho) \backslash \Gamma^{u} \subseteq \tilde{\partial}_{\star} S(\rho)$ (essentially), and (44) holds again, but in a weaker sense.

However, we do not have any result of regularity for $S(\rho)$. So that we do not know in general whether some weak form of (44) holds, or whether there might exist some finite-compliance repartition $\rho$ with a liquid-void interface, that would in this case essentially be included in $\overline{\tilde{\partial}_{\star} S(\rho)}$.

In dimension $N=2$, however, we have the following technical lemma, shown in Appendix C:

Lemma 5.1. Consider $\Omega \subseteq \mathbb{R}^{2}$ an open domain in the plane and a vector field $v \in L_{\text {loc }}^{1}\left(\Omega, \mathbb{R}^{2}\right)$ such that the distributional divergence $\operatorname{div} v$ of $v$ is a bounded Radon measure in $\Omega$. Assume that $v$ vanishes outside of the set 
$S \subseteq \Omega$ of finite perimeter in $\Omega$. Let $S^{0}$ be the set of points where $S$ has density 0 with respect to the Lebesgue measure:

$$
S^{0}=\left\{x \in \Omega: \lim _{\rho \rightarrow 0} \frac{|S \cap B(x, \rho)|}{\rho^{2}}=0\right\}
$$

and $\hat{S}^{0}$ the set of points of $S^{0}$ where the boundary of $S$ has zero 1-dimensional density:

$$
\hat{S}^{0}=\left\{x \in S^{0}: \lim _{\rho \rightarrow 0} \frac{\left|D \mathbf{1}_{S}\right|(B(x, \rho))}{\rho}=0\right\}
$$

Then the measure $\operatorname{div} v$ is carried by the complement of $\hat{S}^{0}$, that is $|\operatorname{div} v|\left(\hat{S}^{0}\right)=0$.

In our case (with little adaptions to study the part $\bar{\partial}_{\star} L(\rho) \cap \partial \Omega$ : we need to extend locally the functions outside of $\Omega$ to apply the lemma), it shows that the measure $\operatorname{div} \sigma_{\rho}$ defined by (43) is essentially carried by $\left(S(\rho)^{0} \cup \tilde{\partial}_{\star} S(\rho)\right) \backslash \Gamma^{u}$, so that we must have $\bar{\partial}_{\star} L(\rho) \backslash \Gamma^{u} \subseteq \tilde{\partial}_{\star} S(\rho)$, and (44) holds in the weak sense (replacing $\partial S(\rho)$ with $\left.\tilde{\partial}_{\star} S(\rho)\right)$.

\section{Appendix A. The $\Gamma$-COnvergence}

We shortly define the $\Gamma$-convergence of functionals (in metric spaces) and its main properties. For more details we refer mainly to [20] and [5].

Given a metric space $(X, d)$ and $F_{k}: X \rightarrow[-\infty,+\infty]$ a sequence of functions, we define for every $u \in X$ the $\Gamma$-liminf of $F$

$$
F^{\prime}(u)=\Gamma-\liminf _{k \rightarrow \infty} F_{k}(u)=\inf _{u_{k} \rightarrow u} \liminf _{k \rightarrow \infty} F_{k}\left(u_{k}\right)
$$

and the $\Gamma$-limsup of $F$

$$
F^{\prime \prime}(u)=\Gamma-\limsup _{k \rightarrow \infty} F_{k}(u)=\inf _{u_{k} \rightarrow u} \limsup _{k \rightarrow \infty} F_{k}\left(u_{k}\right),
$$

and in the case where $F^{\prime}=F^{\prime \prime}$, we let $F=F^{\prime}=F^{\prime \prime}$ and we say that that $F_{k} \Gamma$-converges to $F: X \rightarrow[-\infty,+\infty]$. The functions $F^{\prime}, F^{\prime \prime}$ (hence also $F$ if it exists) are lower semi-continuous on $X$. We have the following two properties:

1. $F_{k} \Gamma$-converges to $F$ if and only if for every $u \in X$,

(i) for every sequence $u_{k}$ converging to $u, F(u) \leq \liminf _{k \rightarrow \infty} F_{k}\left(u_{k}\right)$;

(ii) there exists a sequence $u_{k}$ converging to $u$, with $\limsup _{k \rightarrow \infty} F_{k}\left(u_{k}\right) \leq F(u)$;

2. if $G: X \rightarrow \mathbb{R}$ is continuous and $F_{k} \Gamma$-converges to $F$, then $F_{k}+G \Gamma$-converges to $F+G$.

The following result is the fundamental property of the $\Gamma$-convergence:

Proposition A.1. Assume $F_{k} \Gamma$-converges to $F$ and for every $k$ let $u_{k}$ be a minimizer of $F_{k}$ over $X$. Then, if the sequence (or a subsequence) $u_{k}$ converges to some $u \in X, u$ is a minimizer for $F$ and $F_{k}\left(u_{k}\right)$ converges to $F(u)$.

In particular, we see that it will always be of great interest to show, together with the $\Gamma$-convergence of a sequence $\left(F_{k}\right)_{k \geq 1}$ of functionals, that any sequence $\left(u_{k}\right)_{k \geq 1}$ of minimizers of $F_{k}$ is precompact in $X$.

Finally, we give the following definition of $\Gamma$-convergence in the case where $\left(F_{h}\right)_{h>0}$ is a family of functionals on $X$ indexed by a continuous parameter $h$ : we say that $F_{h} \Gamma$-converges to $F$ in $X$ as $h \downarrow 0$ if and only if for every sequence $\left(h_{j}\right)$ that converges to zero as $j \rightarrow \infty, F_{h_{j}} \Gamma$-converges to $F$. 


\section{Appendix B. The relaxation of $\Lambda(S, L, V)$}

In this section we show why $\bar{\Lambda}$, defined in (6), is the lower semicontinuous envelope in $L^{1}(\Omega)$ of the function $\Lambda$ of equation (4). As a consequence, it will also show how the sequence $\left(\rho^{n}\right)_{n \geq 1}$ in the proof (p. 37) of Theorem 3.1 may be built in a rigorous way. It relies on the following general result:

Lemma B.1. Let $\Omega \subset \mathbb{R}^{N}$ be a Lipschitz-regular bounded open set, and let $\Omega_{j}$ be an increasing sequence of Lipschitz-regular subsets of $\Omega$ such that $\cup_{j \uparrow \infty} \Omega_{j}=\Omega$. Then the three following conditions are equivalent:

(i) $\lim _{j \rightarrow \infty} \mathcal{H}^{N-1}\left(\partial \Omega_{j}\right)=\mathcal{H}^{N-1}(\partial \Omega)$;

(ii) $\forall u \in B V(\Omega) \cap L^{\infty}(\Omega), \lim _{j \rightarrow \infty} \int_{\partial \Omega_{j}} \operatorname{Tr}_{\Omega_{j}} u(x) \mathrm{d} \mathcal{H}^{N-1}(x)=\int_{\partial \Omega} \operatorname{Tr}_{\Omega} u(x) \mathrm{d} \mathcal{H}^{N-1}(x)$;

(iii) $\forall u \in B V(\Omega) \cap L^{\infty}(\Omega), \operatorname{Tr}_{\Omega_{j}} u \mathrm{~d} \mathcal{H}^{N-1}\left\llcorner\partial \Omega_{j} \rightarrow \operatorname{Tr}_{\Omega} u d \mathcal{H}^{N-1}\left\llcorner\partial \Omega\right.\right.$ weakly-* in $\mathcal{M}\left(\Omega^{\prime}\right)$ as $j \rightarrow \infty$, where $\Omega^{\prime}$ is any open set such that $\Omega^{\prime} \supset \supset \Omega$.

Here $\operatorname{Tr}_{\Omega} u$ denotes the trace of $u$ on $\partial \Omega\left(\operatorname{Tr}_{\Omega} u \in L^{1}(\partial \Omega)\right)$ while $\operatorname{Tr}_{\Omega_{j}} u$ denotes the trace of the restriction $\left.u\right|_{\Omega_{j}}$ on $\partial \Omega_{j}$. The assumption that $\partial \Omega_{j}$ is Lipschitz-regular is not absolutely essential: the result still holds if for every $j, \Omega_{j}$ is the intersection of $\Omega$ with some other Lipschitz-regular open subset of $\mathbb{R}^{N}$.

Proof. Clearly (iii) $\Rightarrow$ (ii) $\Rightarrow$ (i). Let us show first that (ii) $\Rightarrow$ (iii). Choose $u \in B V(\Omega)$ and let $\varphi \in$ $C^{1}\left(\Omega^{\prime} ; \mathbb{R}^{N}\right)$ with compact support. Then

$$
\int_{\Omega_{j}} u \operatorname{div} \varphi \mathrm{d} x=\int_{\partial \Omega_{j}} \operatorname{Tr}_{\Omega_{j}} u \varphi \cdot \nu_{\Omega_{j}} \mathrm{~d} \mathcal{H}^{N-1}-\int_{\Omega_{j}} \varphi \cdot D u .
$$

Since $\cap_{j \uparrow \infty}\left(\Omega \backslash \Omega_{j}\right)=\emptyset$, the last term goes to $\int_{\Omega} \varphi \cdot D u$ as $j \rightarrow \infty$ while the left-hand term goes to $\int_{\Omega} u \operatorname{div} \varphi \mathrm{d} x$. It yields

$$
\lim _{j \rightarrow \infty} \int_{\partial \Omega_{j}} \operatorname{Tr}_{\Omega_{j}} u \varphi \cdot \nu_{\Omega_{j}} \mathrm{~d} \mathcal{H}^{N-1}=\int_{\partial \Omega} \operatorname{Tr}_{\Omega} u \varphi \cdot \nu_{\Omega} \mathrm{d} \mathcal{H}^{N-1}
$$

We deduce that $\int_{A \cap \partial \Omega}\left|\operatorname{Tr}_{\Omega} u\right| \mathrm{d} \mathcal{H}^{N-1} \leq \liminf _{j \rightarrow \infty} \int_{A \cap \partial \Omega_{j}}\left|\operatorname{Tr}_{\Omega_{j}} u\right| \mathrm{d} \mathcal{H}^{N-1}$ for every open $A \subset \Omega^{\prime}$. If $u$ is positive and $\mathcal{H}^{N-1}(\partial \Omega \cap A)=0$, we thus get that

$$
\begin{aligned}
\int_{\partial \Omega} \operatorname{Tr}_{\Omega} u \mathrm{~d} \mathcal{H}^{N-1} & =\int_{\partial \Omega \cap A} \operatorname{Tr}_{\Omega} u \mathrm{~d} \mathcal{H}^{N-1}+\int_{\partial \Omega \backslash \bar{A}} \operatorname{Tr}_{\Omega} u \mathrm{~d} \mathcal{H}^{N-1} \\
& \leq \liminf _{j \rightarrow \infty} \int_{\partial \Omega_{j} \cap A} \operatorname{Tr}_{\Omega_{j}} u \mathrm{~d} \mathcal{H}^{N-1}+\liminf _{j \rightarrow \infty} \int_{\partial \Omega_{j} \backslash \bar{A}} \operatorname{Tr}_{\Omega_{j}} u \mathrm{~d} \mathcal{H}^{N-1} \\
& \leq \limsup _{j \rightarrow \infty} \int_{\partial \Omega_{j} \cap A} \operatorname{Tr}_{\Omega_{j}} u \mathrm{~d} \mathcal{H}^{N-1}+\limsup _{j \rightarrow \infty} \int_{\partial \Omega_{j} \backslash \bar{A}} \operatorname{Tr}_{\Omega_{j}} u \mathrm{~d} \mathcal{H}^{N-1} \\
& \leq \limsup _{j \rightarrow \infty} \int_{\partial \Omega_{j}} \operatorname{Tr}_{\Omega_{j}} u \mathrm{~d} \mathcal{H}^{N-1} .
\end{aligned}
$$

If (ii) holds, we see that all inequalities are in fact equalities and we get

$$
\lim _{j \rightarrow \infty} \int_{\partial \Omega_{j} \cap A} \operatorname{Tr}_{\Omega_{j}} u \mathrm{~d} \mathcal{H}^{N-1}=\int_{\partial \Omega \cap A} \operatorname{Tr}_{\Omega} u \mathrm{~d} \mathcal{H}^{N-1} .
$$

We deduce that (iii) also holds for $u$. If $u$ is not positive, then it is enough to show (iii) for the positive and negative parts $u^{+}$and $u^{-}$of $u$ and then take the difference. 
We now show $(i) \Rightarrow($ ii). It is enough to prove (ii) for characteristic functions of finite-perimeter sets. Indeed, if $u_{n}=\sum_{i=1}^{n} \alpha_{i} \mathbf{1}_{E_{i}} \leq u$ ( $u$ is bounded), where $E_{i}$ are finite-perimeter sets, then if (ii) holds for each $\mathbf{1}_{E_{i}}$,

$\int_{\partial \Omega} \operatorname{Tr}_{\Omega} u_{n} \mathrm{~d} \mathcal{H}^{N-1}=\sum_{i=1}^{n} \alpha_{i} \int_{\partial \Omega} \operatorname{Tr}_{\Omega} \mathbf{1}_{E_{i}} \mathrm{~d} \mathcal{H}^{N-1}=\lim _{j \rightarrow \infty} \sum_{i=1}^{n} \alpha_{i} \int_{\partial \Omega_{j}} \operatorname{Tr}_{\Omega_{j}} \mathbf{1}_{E_{i}} \mathrm{~d} \mathcal{H}^{N-1} \leq \liminf _{j \rightarrow \infty} \int_{\partial \Omega_{j}} \operatorname{Tr}_{\Omega_{j}} u \mathrm{~d} \mathcal{H}^{N-1}$.

Since we can assume that, as $n \rightarrow \infty, u_{n} \rightarrow u$ not only in $L^{1}(\Omega)$, but also in $L^{1}(\partial \Omega)$, we get $\int_{\partial \Omega} \operatorname{Tr}_{\Omega} u \mathrm{~d} \mathcal{H}^{N-1} \leq$ $\liminf _{j \rightarrow \infty} \int_{\partial \Omega_{j}} \operatorname{Tr}_{\Omega_{j}} u \mathrm{~d} \mathcal{H}^{N-1}$. Changing $u$ into $-u$, we also have lim $\sup _{j \rightarrow \infty} \int_{\partial \Omega_{j}} \operatorname{Tr}_{\Omega_{j}} u \mathrm{~d} \mathcal{H}^{N-1} \leq \int_{\partial \Omega} \operatorname{Tr}_{\Omega} u \mathrm{~d}$ $\mathcal{H}^{N-1}$, so that (ii) holds for $u$.

Given a finite-perimeter set $E$ in $\Omega$, let $E_{j}=E \cap \Omega_{j}$. First of all, $\lim _{j \rightarrow \infty} \mathbf{1}_{E_{j}}=\mathbf{1}_{E}$ in $L^{1}\left(\Omega^{\prime}\right)$ so that $\left|D \mathbf{1}_{E}\right|\left(\Omega^{\prime}\right) \leq \liminf _{j \rightarrow \infty}\left|D \mathbf{1}_{E_{j}}\right|\left(\Omega^{\prime}\right)$. This may be written

$$
\left|D \mathbf{1}_{E}\right|(\Omega)+\left|D \mathbf{1}_{E}\right|(\partial \Omega) \leq \liminf _{j \rightarrow \infty}\left|D \mathbf{1}_{E}\right|\left(\Omega_{j}\right)+\left|D \mathbf{1}_{E_{j}}\right|\left(\partial \Omega_{j}\right)
$$

but since $\cup_{j \uparrow \infty} \Omega_{j}=\Omega, \lim _{j \rightarrow \infty}\left|D \mathbf{1}_{E}\right|\left(\Omega_{j}\right)=\left|D \mathbf{1}_{E}\right|(\Omega)$, so that

$$
\left|D \mathbf{1}_{E}\right|(\partial \Omega) \leq \liminf _{j \rightarrow \infty}\left|D \mathbf{1}_{E_{j}}\right|\left(\partial \Omega_{j}\right)
$$

In particular if we let $E^{\prime}=\Omega \backslash E$ and $E_{j}^{\prime}=E^{\prime} \cap \Omega_{j}=\Omega_{j} \backslash E$, and still view $D \mathbf{1}_{E}$ and $D \mathbf{1}_{E^{\prime}}$ as measures in $\mathcal{M}\left(\Omega^{\prime}\right)$

$$
\begin{aligned}
& \mathcal{H}^{N-1}(\partial \Omega)=\int_{\partial \Omega} \operatorname{Tr}_{\Omega} \mathbf{1}_{E}+\operatorname{Tr}_{\Omega} \mathbf{1}_{E^{\prime}} \mathrm{d} \mathcal{H}^{N-1}=\left|D \mathbf{1}_{E}\right|(\partial \Omega)+\left|D \mathbf{1}_{E^{\prime}}\right|(\partial \Omega) \\
& \leq \liminf _{j \rightarrow \infty}\left|D \mathbf{1}_{E_{j}}\right|\left(\partial \Omega_{j}\right)+\liminf _{j \rightarrow \infty}\left|D \mathbf{1}_{E_{j}^{\prime}}\right|\left(\partial \Omega_{j}\right) \\
& \leq \limsup _{j \rightarrow \infty}\left|D \mathbf{1}_{E_{j}}\right|\left(\partial \Omega_{j}\right)+\limsup _{j \rightarrow \infty}\left|D \mathbf{1}_{E_{j}^{\prime}}\right|\left(\partial \Omega_{j}\right) \\
& \leq \limsup _{j \rightarrow \infty} \mathcal{H}^{N-1}\left(\partial \Omega_{j}\right)
\end{aligned}
$$

and assuming (i), we deduce that $\lim _{j \rightarrow \infty}\left|D \mathbf{1}_{E_{j}}\right|\left(\partial \Omega_{j}\right)=\left|D \mathbf{1}_{E}\right|(\partial \Omega)$. Since we have $\left|D \mathbf{1}_{E_{j}}\right|\left(\partial \Omega_{j}\right)=\int_{\partial \Omega_{j}} \operatorname{Tr}_{\Omega_{j}} \mathbf{1}_{E}$ $\mathrm{d} \mathcal{H}^{N-1}$, as well as $\left|D \mathbf{1}_{E}\right|(\partial \Omega)=\int_{\partial \Omega} \operatorname{Tr}_{\Omega} \mathbf{1}_{E} \mathrm{~d} \mathcal{H}^{N-1}$, it shows the result for the case $u=\mathbf{1}_{E}$ and Lemma B.1 is proved.

Given $S, L, V$ a partition of $\Omega$, let now $\Lambda(S, L, V)$ be defined by (4), i.e., $\Lambda(S, L, V)=\mathcal{H}^{N-1}(\partial L \cap \Omega)+$ $\mathcal{H}^{N-1}(\partial V \cap \Omega)$, if $\partial L \cap \Omega$ and $\partial V \cap \Omega$ are smooth and $\Gamma_{S} \subset \bar{S}, \Gamma_{L} \subset \bar{S}, \Gamma_{V} \subset \bar{S}$, and $\Lambda(S, L, V)=+\infty$ otherwise. Let also $\bar{\Lambda}$ be defined by (6). Clearly, $\bar{\Lambda}$ is lower semicontinuous (in the $L^{1}(\Omega)$ topology) and since $\bar{\Lambda}(S, L, V)=\Lambda(S, L, V)$ when $\Lambda(S, L, V)<+\infty$, it is clearly less than the l.s.c. envelope of $\Lambda$. To show that it is equal, we must find, given $S, L, V$ a finite-perimeter partition of $\Omega$, a sequence of smooth partitions $S_{j}, L_{j}, V_{j}$ such that $\mathbf{1}_{S_{j}} \rightarrow \mathbf{1}_{S}, \mathbf{1}_{L_{j}} \rightarrow \mathbf{1}_{L}$ in $L^{1}(\Omega)$ and $\limsup _{j \rightarrow \infty} \Lambda\left(S_{j}, L_{j}, V_{j}\right) \leq \bar{\Lambda}(S, L, V)$.

We recall that $\Gamma^{\rho}$ is the closed set $\Gamma_{S} \cup \Gamma_{L} \cup \Gamma_{V}$. Let $d: \Omega \rightarrow \mathbb{R}_{+}$be the distance function $\operatorname{dist}\left(\cdot, \Gamma^{\rho}\right)$. Since $\operatorname{dist}\left(\Gamma_{S}, \Gamma_{L}\right)>0, \operatorname{dist}\left(\Gamma_{L}, \Gamma_{V}\right)>0, \operatorname{dist}\left(\Gamma_{V}, \Gamma_{S}\right)>0$, and since $(c f .[25]$ Th. 3.2.39)

$$
\mathcal{H}^{N-1}\left(\Gamma^{\rho}\right)=\lim _{\delta \rightarrow 0} \frac{|\{x \in \Omega: d(x)<\delta\}|}{\delta}=\lim _{\delta \rightarrow 0} \frac{1}{\delta} \int_{0}^{\delta} \mathcal{H}^{N-1}(\{d=s\}) \mathrm{d} s,
$$

we can choose a sequence $s_{j} \downarrow 0$ such that $\lim _{j \rightarrow \infty} \mathcal{H}^{N-1}\left(\left\{d=s_{j}\right\}\right)=\mathcal{H}^{N-1}\left(\Gamma^{\rho}\right)$, and such that the open sets $\left\{\operatorname{dist}\left(\cdot, \Gamma_{S}\right)<s_{j}\right\},\left\{\operatorname{dist}\left(\cdot, \Gamma_{L}\right)<s_{j}\right\},\left\{\operatorname{dist}\left(\cdot, \Gamma_{V}\right)<s_{j}\right\}$ are Lipschitz-regular and disjoint. Let $\Omega_{j}=\Omega \backslash\{d \leq$ 
$\left.s_{j}\right\}$, and define

$$
\begin{aligned}
& S_{j}=\left(S \cap \Omega_{j}\right) \cup\left\{x \in \Omega: \operatorname{dist}\left(x, \Gamma_{S}\right)<s_{j}\right\}, \\
& L_{j}=\left(L \cap \Omega_{j}\right) \cup\left\{x \in \Omega: \operatorname{dist}\left(x, \Gamma_{L}\right)<s_{j}\right\}, \text { and } \\
& V_{j}=\left(V \cap \Omega_{j}\right) \cup\left\{x \in \Omega: \operatorname{dist}\left(x, \Gamma_{V}\right)<s_{j}\right\} .
\end{aligned}
$$

Then, the fact that $\lim _{j \rightarrow \infty} \bar{\Lambda}\left(S_{j}, L_{j}, V_{j}\right)=\bar{\Lambda}(S, L, V)$ comes from a straightforward application of Lemma B.1. Moreover, it is clear that $\bar{\Lambda}\left(S_{j}, L_{j}, V_{j}\right)=\mathcal{H}^{N-1}\left(\partial_{\star} L_{j}\right)+\mathcal{H}^{N-1}\left(\partial_{\star} V_{j}\right)=\left|D \mathbf{1}_{L_{j}}\right|(\Omega)+\left|D \mathbf{1}_{V_{j}}\right|(\Omega)$.

By standard mollifying methods we can modify slightly for every $j$ the partition $S_{j}, L_{j}, V_{j}$ into a smooth partition $S_{j}^{\prime}, L_{j}^{\prime}, V_{j}^{\prime}$, conserving the properties that $\Gamma_{S} \subset \overline{S_{j}^{\prime}}$, etc., that $\mathbf{1}_{S_{j}^{\prime}} \rightarrow \mathbf{1}_{S}$ in $L^{1}(\Omega)$ as $j \rightarrow \infty$, etc, and not changing too much the perimeters so that we still have $\lim _{j \rightarrow \infty} \Lambda\left(S_{j}^{\prime}, L_{j}^{\prime}, V_{j}^{\prime}\right)=\lim _{j \rightarrow \infty} \bar{\Lambda}\left(S_{j}^{\prime}, L_{j}^{\prime}, V_{j}^{\prime}\right)$ $=\bar{\Lambda}(S, L, V)$.

\section{Appendix C. Proof of Lemma 5.1}

In this appendix we give the proof of Lemma 5.1. We know that we can write $\operatorname{div} v=g|\operatorname{div} v|$ where $g$ is the Radon-Nykodym derivative of $\operatorname{div} v$ with respect to its variation $|\operatorname{div} v|$, and is given $|\operatorname{div} v|$-a.e. in $\Omega$ by

$$
g(x)=\lim _{\rho \rightarrow 0} \frac{\operatorname{div} v(B(x, \rho))}{|\operatorname{div} v|(B(x, \rho))} .
$$

All we have to show is that if $x$ is a point in $\hat{S}^{0}$ such that this equality holds, then $g(x)=0$. In order to do this we will show there exists a sequence $\left(\rho_{n}\right)_{n \geq 1}$ of radii that go to zero as $n \rightarrow \infty$, such that for every $n$, $\operatorname{div} v\left(B\left(x, \rho_{n}\right)\right)=0$.

In the following $x$ is fixed and we will denote by $B_{\rho}$ the ball $B(x, \rho)$. Our first claim is that for almost every radius $\rho>0$ (small), $\operatorname{div} v\left(B_{\rho}\right)=\int_{\partial B_{\rho}} v_{r}(y) \mathrm{d} \mathcal{H}^{1}(y)$ where $v_{r}$ denotes the radial component $v \cdot e_{r}\left(e_{r}=\right.$ $(y-x) /|y-x|)$ of the field $v$.

For a given $\rho>0$ and every small $\alpha>0$, we introduce the function

$$
\psi_{\alpha}(y)= \begin{cases}1 & \text { if } y \in B_{\rho-\alpha} \\ \frac{\rho-r}{\alpha} & \text { if } r=|y-x| \in(\rho-\alpha, \rho) \\ 0 & \text { if } y \notin B_{\rho} .\end{cases}
$$

Then,

$$
\begin{aligned}
\operatorname{div} v\left(B_{\rho}\right) & =\lim _{\alpha \rightarrow 0} \int_{\Omega} \psi_{\alpha}(y) d(\operatorname{div} v)(y)=\lim _{\alpha \rightarrow 0}-\int_{\Omega} \nabla \psi_{\alpha}(y) v(y) \mathrm{d} y \\
& =\lim _{\alpha \rightarrow 0} \frac{1}{\alpha} \int_{B_{\rho} \backslash B_{\rho-\alpha}} v_{r}(y) \mathrm{d} y=\lim _{\alpha \rightarrow 0} \frac{1}{\alpha} \int_{\rho-\alpha}^{\rho} \mathrm{d} r \int_{\partial B_{r}} v_{r}(y) \mathrm{d} \mathcal{H}^{1}(y) .
\end{aligned}
$$

Fix $\rho_{0}$ small (such that $B_{\rho_{0}} \subset \Omega$ ) and define for $0<r<\rho_{0}$ the function $f(r)=\int_{\partial B_{r}} v_{r} \mathrm{~d} \mathcal{H}^{1}$. Since $v \in L^{1}\left(B_{\rho_{0}}\right)$, $f \in L^{1}\left(0, \rho_{0}\right)$ so that for almost every $\rho \in\left(0, \rho_{0}\right)$,

$$
\lim _{\alpha \rightarrow 0} \frac{1}{\alpha} \int_{\rho-\alpha}^{\rho} f(r) \mathrm{d} r=f(\rho) .
$$

We deduce that for a.e. $\rho \in\left(0, \rho_{0}\right), \operatorname{div} v\left(B_{\rho}\right)=f(\rho)=\int_{\partial B_{\rho}} v_{r} \mathrm{~d} \mathcal{H}^{1}$ which proves the first claim.

Now, we show that for every small $\rho_{0}>0$, the set of radii $\rho \in\left(0, \rho_{0}\right)$ such that $\partial B_{\rho} \cap S=\emptyset$ (essentially) has positive measure. Notice that for such a $\rho, v=0 \mathcal{H}^{1}$-a.e. in $\partial B_{\rho}$ (by assumption), so that $\int_{\partial B_{\rho}} v_{r} \mathrm{~d} \mathcal{H}^{1}=0$. 
Therefore if this second claim is true, then the existence of a sequence $\left(\rho_{n}\right)$ such that $\rho_{n} \rightarrow 0$ as $n \rightarrow \infty$ and $\operatorname{div} v\left(B_{\rho_{n}}\right)=0$ becomes obvious, and the proof of the lemma is achieved.

Choose some $\delta>0$ and assume that $\rho_{0}$ has been chosen small enough in order to have (we use the fact here that $\left.x \in \hat{S}^{0}\right)$

and

$$
\left|S \cap B_{\rho_{0}}\right| \leq \delta \rho_{0}^{2}
$$

$$
\left|D \mathbf{1}_{S}\right|\left(B_{\rho_{0}}\right) \leq \delta \rho_{0}
$$

We let $I_{1}=\left\{r \in\left(\rho_{0} / 2, \rho_{0}\right): \mathcal{H}^{1}\left(\partial B_{r} \backslash S\right)=0\right\}, I_{2}=\left\{r \in\left(\rho_{0} / 2, \rho_{0}\right): \mathcal{H}^{1}\left(S \cap \partial B_{r}\right)<\mathcal{H}^{1}\left(\partial B_{r}\right)\right\}$, and $I=I_{1} \cup I_{2}$. Notice that the complement of $I$ in $\left(\rho_{0} / 2, \rho_{0}\right)$ is the set of radii $r$ such that $\mathcal{H}^{1}\left(\partial B_{r} \cap S\right)=0$, i.e., such that the intersection $\partial B_{r} \cap S$ is essentially empty and therefore $\int_{\partial B_{r}} v_{r} \mathrm{~d} \mathcal{H}^{1}=0$. We thus have to show that $|I|<\rho_{0} / 2$.

First,

so that $\left|I_{1}\right| \leq \delta \rho_{0} / \pi$. Then,

$$
\left|S \cap B_{\rho_{0}}\right| \geq \int_{I_{1}} 2 \pi r \mathrm{~d} r \geq \pi \rho_{0}\left|I_{1}\right|
$$

$$
\left|D \mathbf{1}_{S}\right|\left(B_{\rho_{0}}\right)=\mathcal{H}^{1}\left(\partial_{\star} S \cap B_{\rho_{0}}\right) \geq \int_{0}^{\rho_{0}} \mathcal{H}^{0}\left(\partial_{\star} S \cap \partial B_{r}\right) \mathrm{d} r
$$

For almost every $r, \partial_{\star} S \cap \partial B_{r}=\partial_{\star}\left(S \cap \partial B_{r}\right)$, and if moreover $r \in I_{2}$, then $\partial_{\star}\left(S \cap \partial B_{r}\right)$ must contain at least two points. Therefore $\left|D \mathbf{1}_{S}\right|\left(B_{\rho_{0}}\right) \geq 2\left|I_{2}\right|$. We deduce that $\left|I_{2}\right| \leq \delta \rho_{0} / 2$, so that if $\delta$ has been chosen strictly smaller than $\pi /(2+\pi),|I|<\rho_{0} / 2$, which is what we needed to show. The proof of the lemma is achieved.

Part of this work was carried out while A. Chambolle was visiting the Department of Mathematics of the Technical University of Denmark, where B. Bourdin was employed, as a research assistant professor. Both author would like to thank M.P. Bendsøe for making this visit possible and for the exciting discussions held there. We would also like to thank R.V. Kohn for his numerous and invaluable comments and advices.

\section{REFERENCES}

[1] G. Alberti, Variational models for phase transitions, an approach via $\Gamma$-convergence, in Calculus of Variations and Partial Differential Equations, edited by G. Buttazzo et al. Springer-Verlag (2000) 95-114.

[2] G. Allaire, É. Bonnetier, G.A. Francfort and F. Jouve, Shape optimization by the homogenization method. Numer. Math. 76 (1997) 27-68.

[3] G. Allaire, Shape optimization by the homogenization method. Springer-Verlag, New York (2002).

[4] L. Ambrosio and G. Buttazzo, An optimal design problem with perimeter penalization. Calc. Var. Partial Differential Equations 1 (1993) 55-69.

[5] H. Attouch, Variational convergence for functions and operators. Applicable Mathematics Series. Pitman (Advanced Publishing Program), Boston, Mass.-London (1984).

[6] S. Baldo, Minimal interface criterion for phase transitions in mixtures of Cahn-Hilliard fluids. Ann. Inst. H. Poincaré Anal. Non Linéaire 7 (1990) 67-90.

[7] A.C. Barroso and I. Fonseca, Anisotropic singular perturbations - the vectorial case. Proc. Roy. Soc. Edinburgh Sect. A 124 (1994) 527-571.

[8] M.P. Bendsøe, Optimization of Structural Topology, Shape and Material. Springer Verlag, Berlin Heidelberg (1995).

[9] M.P. Bendsøe and O. Sigmund, Material interpolation schemes in topology optimization. Arch. Appl. Mech. 69 (1999) 635-654.

[10] É. Bonnetier and A. Chambolle, Computing the equilibrium configuration of epitaxially strained crystalline films. SIAM J. Appl. Math. 62 (2002) 1093-1121.

[11] B. Bourdin and A. Chambolle, Implementation of an adaptive finite-element approximation of the Mumford-Shah functional. Numer. Math. 85 (2000) 609-646.

[12] B. Bourdin, G.A. Francfort and J.-J. Marigo, Numerical experiments in revisited brittle fracture. J. Mech. Phys. Solids 48 (2000) 797-826.

[13] J.W. Cahn and J.E. Hilliard, Free energy of a nonuniform system I - interfacial free energy. J. Chem. Phys. 28 (1958) $258-267$.

[14] A. Chambolle, Finite-differences discretizations of the Mumford-Shah functional. ESAIM: M2AN 33 (1999) 261-288. 
[15] B.-C. Chen and N. Kikuchi, Topology optimization with design-dependent loads. Finite Elem. Anal. Des. 37 (2001) 57-70.

[16] L.Q. Chen and J. Shen, Application of semi implicit Fourier-spectral method to phase field equations. Comput. Phys. Comm. 108 (1998) 147-158.

[17] A. Cherkaev, Variational methods for structural optimization. Springer-Verlag, New York (2000).

[18] A. Cherkaev and R.V. Kohn, Topics in the mathematical modelling of composite materials. Birkhäuser Boston Inc., Boston, MA (1997).

[19] P.G. Ciarlet, Mathematical elasticity. Vol. I. North-Holland Publishing Co., Amsterdam (1988). Three-dimensional elasticity.

[20] G. Dal Maso, An introduction to $\Gamma$-convergence. Birkhäuser, Boston (1993).

[21] I. Ekeland and R. Témam, Convex analysis and variational problems. Society for Industrial and Applied Mathematics (SIAM), Philadelphia, PA, English Edition (1999). Translated from the French.

[22] L.C. Evans and R.F. Gariepy, Measure theory and fine properties of functions. CRC Press, Boca Raton, FL (1992).

[23] D. Eyre, Systems of Cahn-Hilliard equations. SIAM J. Appl. Math. 53 (1993) 1686-1712.

[24] K.J. Falconer, The geometry of fractal sets. Cambridge University Press, Cambridge (1986).

[25] H. Federer, Geometric measure theory. Springer-Verlag, New York (1969).

[26] E. Giusti, Minimal surfaces and functions of bounded variation. Birkhäuser, Boston (1984).

[27] R.B. Haber, C.S. Jog and M.P. Bendsøe, A new approach to variable-topology shape design using a constraint on the perimeter. Struct. Optim. 11 (1996) 1-12.

[28] V.B. Hammer and N. Olhoff, Topology optimization of continuum structures subjected to pressure loading. Struct. Multidisc. Optim. 19 (2000) 85-92.

[29] R.V. Kohn and G. Strang, Optimal design and relaxation of variational problems I-III. Comm. Pure Appl. Math. 39 (1986) 113-137, 139-182, 353-377.

[30] R.V. Kohn and G. Strang, Optimal design in elasticity and plasticity. Internat. J. Numer. Methods Engrg. 22 (1986) $183-188$.

[31] P.H. Leo, J.S Lowengrub and H.J. Jou, A diffuse interface model for microstructural evolution in elastically stressed solids. Acta Mater. 46 (1998) 2113-2130.

[32] L. Modica and S. Mortola. Il limite nella $\Gamma$-convergenza di una famiglia di funzionali ellittici. Boll. Un. Mat. Ital. A (5) 14 (1977) 526-529.

[33] L. Modica and S. Mortola, Un esempio di $\Gamma^{-}$-convergenza. Boll. Un. Mat. Ital. B (5) 14 (1977) 285-299.

[34] M. Negri, The anisotropy introduced by the mesh in the finite element approximation of the Mumford-Shah functional. Numer. Funct. Anal. Optim. 20 (1999) 957-982.

[35] R.H. Nochetto, S. Rovida, M. Paolini and C. Verdi, Variational approximation of the geometric motion of fronts, in Motion by mean curvature and related topics (Trento, 1992) de Gruyter, Berlin (1994) 124-149.

[36] S.J. Osher and F. Santosa, Level set methods for optimization problems involving geometry and constraints. I. Frequencies of a two-density inhomogeneous drum. J. Comput. Phys. 171 (2001) 272-288.

[37] M. Paolini and C. Verdi, Asympto. and numerical analyses of the mean curvature flow with a space-dependent relaxation parameter. Asymptot. Anal. 5 (1992) 553-574.

[38] J.A. Sethian and A. Wiegmann, Structural boundary design via level set and immersed interface methods. J. Comput. Phys. 163 (2000) 489-528.

[39] R. Temam, Problèmes mathématiques en plasticité. Gauthier-Villars, Paris (1983).

[40] W.P. Ziemer, Weakly Differentiable Functions. Springer-Verlag, Berlin (1989). 